\title{
SPACE-TIME PROCESSES, PARABOLIC FUNCTIONS AND ONE-DIMENSIONAL DIFFUSIONS ${ }^{\left({ }^{1}\right)}$
}

\author{
BY
}

\section{TZE LEUNG LAI}

\begin{abstract}
In this paper, we study the properties of the space-time process and of parabolic functions associated with a Markov process. Making use of these properties and the asymptotic behavior of the first passage probabilities near the boundary points, we prove certain theorems concerning when $u(X(t), t)$ is a martingale, where $X(t)$ is a conservative regular one-dimensional diffusion with inaccessible boundaries. A characterization of the class of parabolic functions associated with classical diffusions is also obtained.
\end{abstract}

1. Introduction. Robbins and Siegmund [10] have made use of the martingale

$$
\int_{0}^{\infty} \exp \left(y W(t)-\frac{t}{2} y^{2}\right) d F(y), \quad t \geq 0
$$

to evaluate the probability that the standard Wiener process $W(t)$ would ever cross certain boundaries which are moving with time. In [8], by making use of martingales of the form $u(X(t), t)$, we extend the Robbins-Siegmund method to find boundary crossing probabilities for other Markov processes $X(t)$, and the question of when $u(X(t), t)$ is a martingale is also considered. As a moving boundary in space is a fixed boundary in space-time, it is natural for us to look at the spacetime process. In $\$ 2$, we shall examine the properties of the space-time process, taking into account the special features of the time variable. The continuity properties of parabolic functions associated with continuous strong Feller processes are also established. Making use of these results, we characterize the class of parabolic functions associated with classical nonstationary diffusions in \$3. Our main interest, however, lies in martingales of the form $u(X(t), t)$ when $X(t)$ is a conservative one-dimensional regular diffusion. Theorem 5 is concerned with the situation when both boundaries are natural, while Theorem 6 deals with entrance boundaries. In $\$ 5$, we give some applications of our results to find certain boundary crossing probabilities for $X(t)$.

Received by the editors November 11, 1971.

AMS (MOS) subject classifications (1969). Primary 6060, 6062.

Key words and phrases. Space-time processes, parabolic functions, characteristic operators, semigroups, infinitesimal generators, martingales, diffusions, inacessible boundaries, entrance boundaries, natural boundaries, scale, positive increasing (decreasing) solutions, boundary crossing probabilities.

(1) This paper is based on part of the author's doctoral dissertation written at Columbia University under the supervision of Professor David O. Siegmund, to whom the author wishes to express his deep gratitude. 
2. Characteristic operators of space-time processes and parabolic functions. Let $Y(t), 0 \leq t<\zeta$, be a Markov process which may not be temporally homogeneous and which is defined up to a lifetime $\zeta \in[0, \infty]$. Let $M$ denote the state space of $Y(t)$, and let $P(s, x ; t, \Gamma)$ be its transition function. It is well known (see for example [4] or [6]) that the corresponding spaceotime process $Z(t)$ is a temporally homogeneous Markov process. The state space of $Z(t)$ is $M \times[0, \infty)$, and its transition function $\bar{P}(t, z, \Lambda)$ with $z=(x, s)$ and $\Lambda$ being any measurable subset of $M \times[0, \infty)$ is determined by

$$
\bar{P}(t,(x, s), \Gamma \times C)= \begin{cases}P(s, x ; s+t, \Gamma) & \text { if } s+t \in C, \\ 0 & \text { otherwise, }\end{cases}
$$

where $\Gamma$ is any measurable subset of $M$ and $C$ is any Borel subset of $[0, \infty)$. Obviously $Z(t)$ is conservative iff $Y(t)$ is conservative, while $Z(t)$ is normal iff $Y(t)$ is normal. (As in [5], we say that the Markov process $Y(t)$ is normal if $\lim _{t ! s} P(s, x ; t, M)=1$ for all $x, s$.)

Let $\vec{B}$ be the Banach space of all bounded measurable functions on $M \times$ $[0, \infty)$. For each $u \in \bar{B},\|u\|$ is defined as $\sup _{x \in M, t>0}|u(x, t)|$ and we define

$$
\begin{aligned}
& \bar{T}_{t} u(x, s)=E[u(Z(t)) \mid Z(0)=(x, s)]=\int u(y, t+s) P(s, x ; t+s, d y), \\
& \bar{G}_{a} u(x, s)=\int_{0}^{\infty} e^{-a t} \bar{T}_{t} u(x, s) d t .
\end{aligned}
$$

Then $\left\{\bar{T}_{t}, t \geq 0\right\}$ is a contraction semigroup and $\bar{G}_{a}, \alpha>0$, is the resolvent. Let $\bar{A}$ be the (strong) infinitesimal generator of the semigroup and its domain be $\mathscr{D}(\bar{A})$. Then for $u \in \mathfrak{T}(\bar{A}), \bar{A} u=0$ implies that $\bar{T}_{t} u=u$ and so $u(Y(t+s), t+s), t \geq 0$, is a martingale with respect to $P_{s, x}$ for any $s, x$. (Here we follow the usual convention by defining $u(Y(t), t)=0$ if $t \geq \zeta$.) The same conclusion is also true if we replace the strong infinitesimal generator $\bar{A}$ by the weak infinitesimal generator of the semigroup $\left\{\bar{T}_{t^{\prime}} t \geq 0\right\}$. We now investigate to what extent the above conclusion carries over when we replace $\bar{A}$ which is global in nature by the characteristic operator U (also known as Dynkin's generator) of the space-time process $Z(t)$.

Let $Z_{T}(t)$ denote the part of $Z(t)$ on $M \times[T, \infty), T \geq 0 . Z_{T}(t)$ is just the space-time process of the process $\{Y(t), T \leq t<\zeta\}$. Assume that $M$ is a locally compact Hausdorff space satisfying the second axiom of countability and that $Y(t)$ has continuous sample paths. We shall say that a real-valued function $u(x, t)$ defined on an open subset $G$ of $M \times[T, \infty)$ is parabolic for the process $Y(t)$ on the set $G$ if it is harmonic for the space-time process $Z_{T}(t)$ on $G$. Note that if $V$ is an open subset of $M$ and $T_{1}>T>0$, then $V \times\left[T, T_{1}\right)$ is open in $M \times[T, \infty)$, but it is not open in $M \times[0, \infty)$ and its boundary in $M \times[0, \infty)$ 
contains $V \times\{T\}$ which is a set of irregular boundary points for the process $Z(t)$. Similarly we define $v: G \rightarrow(-\infty, \infty]$ to be superparabolic for $Y(t)$ on $G$ if it is superharmonic for $Z_{T}(t)$ on $G$. (We say that a function $f: D \rightarrow(-\infty, \infty]$ is superharmonic for a temporally homogeneous continuous Markov process $X(t)$ on an open subset $D$ of the state space which is a locally compact, second countable, Hausdorff space if the function $f$ is nearly Borel, continuous in the intrinsic topology, and given any open subset $U$ with compact closure contained in $D, f$ is bounded below on $U$ and $E_{x} f\left(X\left(\tau_{U}\right)\right) \leq f(x)$ for any $x \in D$, where $\tau_{U}$ denotes the first exit time from $U$. If both $f$ and $-f$ are superharmonic for $X(t)$ on $D$, then we say that $f$ is harmonic for $X(t)$ on $D$. For further properties of harmonic and superharmonic functions, see Chapter 12 of [5].) Let $u$ be superparabolic for $Y(t)$ on $G$, and let $U$ be any open subset of $M \times[T, \infty)$ with compact closure contained in $G$. It is easy to see that $u\left(Y\left(\left(t \wedge \tau_{U}\right)+s\right),\left(t \wedge \tau_{U}\right)+s\right), t \geq 0$, is a supermartingale with respect to $P_{s, x}$ for any $(x, s) \in G$, where $\tau_{U}$ is the first exit time from $U$ by $Z(t)$.

Suppose $Y(t), 0 \leq t<\zeta$, is a continuous Markov process on $M$ and $Z(t), t \geq$ 0 , is its space-time process. Then $Z(t)$ is a continuous Markov process and so its characteristic operator $\mathcal{U}$ obeys the minimum principle (cf. [5]). In fact, it obeys a stronger form of the minimum principle: Let $G$ be an open subset of $M \times[0, \infty)$ and $\left(x_{0}, t_{0}\right) \in G$. Let $f$ be a real-valued function on $G$ such that $\mathrm{U}_{f}\left(x_{0}, t_{0}\right)$ exists. If $f$ attains its minimum on $G \cap$ $\left(M \times\left[t_{0}, \infty\right)\right)$ at $\left(x_{0}, t_{0}\right)$ and $f\left(x_{0}, t_{0}\right) \leq 0$, then $\mathcal{U}_{f}\left(x_{0}, t_{0}\right) \geq 0$. (Compare the classical maximal principles for parabolic and elliptic operators.)

By the definition of the characteristic operator $\mathcal{U}$, in order that $f(x, t)$ is parabolic on $G$, it is necessary that $\mathcal{U} f(x, t)=0$ for all $(x, t) \in G$. To what extent is the condition $U_{f}=0$ on $G$ also sufficient? Dynkin [5] has proved a theorem relating continuous harmonic functions on an open set $G$ for a continuous standard process to continuous solutions of $\mathcal{U}_{f}=0$ on $G$. One of the conditions in his theorem is that the processes stopped at the first exit times from certain open sets with compact closures contained in $G$ are Feller processes. This condition, however, often fails for space-time processes. The following theorem is a modification of Dynkin's result, taking into consideration the special features of the time variable.

Theorem 1. Let $\left(Y(t), \zeta, \mathfrak{F}_{t}^{s}, P_{s, x}\right)$ be a normal strong Markov process with continuous sample paths, lifetime $\zeta$ and state space $M$ which is a locally compact Hausdorff space satisfying the second axiom of countability. Let $\mathcal{U}$ denote the characteristic operator of the corresponding space-time process $Z(t)$. Let $G$ be an open subset of $M \times[T, \infty)(T \geq 0)$. Suppose that given any open subset $U$ of $M \times[T, \infty)$ with compact closure contained in $G$, there exist $t_{n}>\cdots>t_{0}=$ $T(n \geq 1)$ and disjoint sets $U_{j}^{i}\left(i=1, \ldots, n ; j=1, \ldots, n_{i}\right)$ such that 
(i) $\widetilde{U}=\bigcup_{i=1}^{n} \bigcup_{j=1}^{n_{i}} U_{j}^{i}$ has a compact closure contained in $G$, and $U \subset \widetilde{U}$.

(ii) $U_{j}^{i}$ is an open subset of $M \times\left[t_{i-1}, t_{i}\right)$ for all $i, j$.

(iii) $Z_{i}\left(t \wedge \tau_{i j}\right)$ is a Feller process for all $i, j$, where $Z_{i}(t)$ denotes the part of $Z(t)$ on $M \times\left[t_{i-1}, \infty\right)$, and $\tau_{i j}=\inf \left\{t \geq 0 \mid Z_{i}(t) \notin U_{j}^{i}\right\}$.

Under these conditions, in order for a continuous function $f: G \rightarrow(-\infty, \infty)$ to be parabolic for $Y(t)$ on the set $G$, it is necessary and sufficient that $f \in \mathscr{D}_{\mathcal{U}}(G)$ and $\mathrm{U} f(x, t)=0$ for all $(x, t) \in G$ (where $\mathfrak{D}_{\mathcal{U}}(G)$ denotes the class of all functions $u$ for which $\mathcal{U}_{u}(x, t)$ exists at every point $\left.(x, t) \in G\right)$.

Proof. The time-homogeneous process $Z(t)$ is a continuous strong Markov normal process on $M \times[0, \infty)$ which is a locally compact, second countable, Hausdorff space. Let $f \in \mathfrak{D}_{\mathcal{U}}(G)$ be a continuous function on $G$ such that $\mathrm{U} f=0$ on $G$. The process $Z_{i}\left(t \wedge \tau_{i j}\right)$ is a Feller process on $M \times\left[t_{i-1}, \infty\right)$. Obviously it is also a $\hat{C}$ process. (As in [5], we say that a temporally homogeneous Markov process $X(t)$ on a locally compact, second countable, Hausdorff space $H$ is a $\hat{C}$ process if, given any $t \geq 0$ and $f \in \hat{C}$, the function $g(x)=E_{x} f(X(t))$ also belongs to $\hat{C}$, where $\hat{C}$ denotes the class of all continuous real-valued functions $f$ on $H$ such that, given $\epsilon>0$, there exists a compact subset $K$ for which $|f(x)|<\epsilon$ if $x \notin K$.) Let $\hat{A}$ denote the $\hat{C}$-infinitesimal generator and $\hat{U}$ the characteristic operator of the process $Z_{i}\left(t \wedge \tau_{i j}\right)$. Then $\hat{A}$ is the restriction of $\hat{U}$ to the class of all functions $g \in \mathscr{D}_{\hat{\mathrm{u}}} \cap \hat{C}$ such that $\hat{U}_{g} \in \hat{C}$. The space $M \times[0, \infty)$ is metrizable, and let us assume that some metric $\rho$ is introduced into it. Let $V$ be any open subset of $M \times\left[t_{i-1}, \infty\right)$ containing $U_{j}^{i}$ such that the closure of $V$ is compact and is contained in $G$. Let $V^{c}$ denote the complement of $V$ in $M \times\left[t_{i-1}, \infty\right)$. We define

$$
\hat{f}(z)= \begin{cases}\frac{\rho\left(z, V^{c}\right)}{\rho\left(z, V^{c}\right)+\rho\left(z, U_{i}^{i}\right)} f(z) & \text { if } z \in V, \\ 0 & \text { if } z \in V^{c} .\end{cases}
$$

Then $\hat{f} \in \mathscr{D}_{\hat{\mathrm{U}}} \cap \hat{C}$ and $\hat{\mathrm{U}} \hat{f}=0$. Hence $\hat{A} \hat{f}=0$. Also $\hat{f}(z)=f(z)$ for all $z \in U_{j}^{i}$. From this it follows that $E_{s, x} f\left(Z_{i}\left(t \wedge \tau_{i j}\right)\right)=f(x, s)$ for all $(x, s) \in U_{j}^{i}$ and $t>0$. Clearly given $(x, s) \in U_{j}^{i}, E_{s, x} f\left(Z\left(t \wedge \tau_{i j}\right)\right)=E_{s, x} f\left(Z\left(\tau_{i j}\right)\right)$ for all large $t$. Therefore, for any $(x, s) \in U_{j}^{i}, E_{s, x} f\left(Z\left(\tau_{i j}\right)\right)=f(x, s)$. This implies that, for all $(x, s) \epsilon$ $\widetilde{U}, E_{s, x} f(Z(\tau \widetilde{U}))=f(x, s)$, as can be easily verified by repeated use of the strong Markov property and conditional expectations. Given an open set $U$ with compact closure contained in $G$, we can find an open set $U$, containing the closure of $U$ such that the closure of $U_{1}$ is a compact subset of $G$. Take $\tilde{U}_{1}$ containing $U_{1}$ and satisfying conditions (i), (ii), (iii). Then $E_{s, x}\left(Z\left(\tau_{\widetilde{U}_{1}}\right)\right)=f(x, s)$ for all $(x, s) \in \widetilde{U}_{1}$. From this, it then follows that $E_{s, x} f\left(Z\left(\tau_{U}\right)\right)=f(x, s)$ for all $(x, s) \in U$, and $f$ is parabolic for $Y(t)$ on G. Q.E.D. 
We say that a Markov process $\left(Y(t), \zeta, \mathcal{F}_{t}^{s}, P_{s, x}\right)$ on a topological measurable space is a strong Feller process if given any bounded measurable function $\phi$ on the state space $M$, the function $F_{T}(x, s)=\int \phi(y) P(s, x ; T, d y)$ is continuous in $(x, s)$ for $s<T$ (cf. [4]). When $Y(t)$ is strong Feller, the space-time process may not be strong Feller. However the strong Feller property of $Y(t)$ may be exploited to investigate properties of parabolic functions on cylinder sets (i.e., sets of the form $\left.V \times\left(t_{1}, t_{2}\right)\right)$, and these properties can then be extended to the case of parabolic functions on an arbitrary open subset $G$ of $M \times[T, \infty)$.

Theorem 2. Let $\left(Y(t), \zeta, \mathcal{F}_{t}^{s}, P_{s, x}\right)$ be a continuous strong Feller process on a metric space $M$. Let $V$ be an open subset of $M$ and $\sigma_{V}^{s}=\inf \{t \geq s \mid Y(t) \notin V\}$. Suppose for any $\epsilon>0, T>0$ and any open set $\Gamma$ with compact closure contained in $V$,

$$
\begin{array}{r}
P\left(s, x ; t, B_{\epsilon}(x)\right) \rightarrow 1 \text { as } t \downarrow s \text { uniformly for } x \in \Gamma \text { and } s \in[0, T], \\
\text { where } B_{\epsilon}(x)=\{y \in M \mid \rho(x, y)<\epsilon\} .
\end{array}
$$

Then given any $T>0$ and any bounded measurable function $f$ on $M \times\left[\begin{array}{ll}0 & \infty\end{array}\right)$, the functions

and

$$
F_{T}(x, s)=E_{s, x} f(Y(T), T) I\left[\sigma_{V}^{s}>T\right]
$$

$$
\Psi_{T}(x, s)=E_{s, x} f\left(Y\left(\sigma_{V}^{s}\right), \sigma_{V}^{s}\right) I_{\left[\sigma_{V}^{s} \leq T\right]}
$$

are continuous in $\left(\begin{array}{ll}x & s\end{array}\right)$ for $x \in V$ and $0 \leq s<T$ where we use $I_{A}$ to denote the indicator function (or commonly called the characteristic function) of the event $A$.

Lemma 1. Let $Y(t), V$ be the same as in Theorem 2. Then for any $\epsilon>0$, $T>0$ and any open set $\Gamma$ with compact closure contained in $V$,

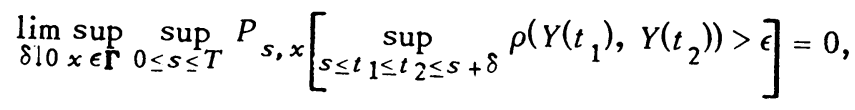

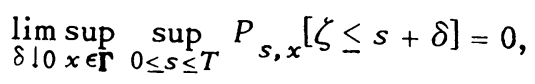

$$
\limsup _{\delta ! 0} \sup _{x \in \Gamma} P_{s, x}\left[\sigma_{V}^{s} \leq s+\delta\right]=0 .
$$

Proof. The proof of (a) is similar to that of Lemma 13.2 in [5], using Lemma 6.6 in [4] in place of the estimate 3.11B in [5]. As to (b), we note that $P_{s, x}[\zeta \leq s+\delta]=1-P(s, x ; s+\delta, M)$ and so (b) follows from (2). If $V=M$, then 
(c) simply reduces to (b). If $V \neq M$, we let $\epsilon=\rho(\Gamma, M-V)$. Then for all $x \in \Gamma$,

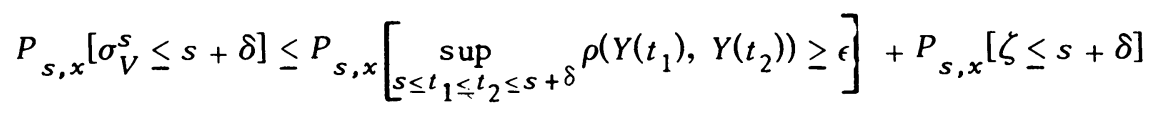

and (c) follows from (a) and (b). Q.E.D.

Proof of Theorem 2. Take $s_{0} \in[0, T), x_{0} \in V$. Take an open neighborhood $\Gamma$ of $x_{0}$ having compact closure contained in $V$. Given $\epsilon>0$, by Lemma 1(c), we can can choose $\delta \in\left(0, T-s_{0}\right)$ such that $P_{s, x}\left[\sigma_{V}^{s} \leq s+2 \delta\right]<\epsilon$ for all $x \in \Gamma$ and $0 \leq s \leq s_{0}+\delta$. Then if $x \in \Gamma, s \in\left(s_{0}-\delta, s_{0}+\delta\right)$, we obtain using the strong Markov property

$$
\begin{aligned}
& \Psi_{T}(x, s)= E_{s, x} f\left(Y\left(\sigma_{V}^{s}\right), \sigma_{V}^{s}\right) I\left[\sigma_{V}^{s} s_{0}+\delta\right] \\
&-E_{s, x} \Psi_{T}\left(Y\left(s_{0}+\delta\right), s_{0}+\delta\right) I \\
& {\left[\sigma_{V}^{s} s_{0} s_{0}+\delta\right] }
\end{aligned}
$$

Since $Y(t)$ is strong Feller, $\int \Psi_{T}\left(y, s_{0}+\delta\right) P\left(s, x ; s_{0}+\delta, d y\right)$ is a continuous function in $(x, s)$ for $s<s_{0}+\delta$. From this, it is then clear that $\Psi_{T}$ is continuous at $\left(x_{0}, s_{0}\right)$. The continuity of $F_{T}$ can be proved similarly. Q.E.D.

Corollary. Let $\left(Y(t), \zeta, \mathcal{F}_{t}^{s}, P_{s, x}\right)$ be a continuous strong Feller process on a metric space such that, for any $\epsilon>0, T>0$ and any open set $\Gamma$ with compact closure contained in $M,(2)$ bolds. Let $Z(t)$ be the corresponding space-time process.

(i) Let $V$ be an open subset of $M$ baving compact closure. Let $f:(\partial V \times$ $\left.\left[s_{1}, s_{2}\right)\right) \cup\left(\bar{V} \times\left\{s_{2}\right\}\right) \rightarrow(-\infty, \infty)$ be a bounded measurable function. For $(x, s) \in$ $\left[s_{1}, s_{2}\right) \times V$, define $u(x, s)=E_{s, x} f\left(Z\left(\tau_{U}\right)\right)$, where $\tau_{U}$ is the first exit time by $Z(t)$ from $U=V \times\left[s_{1}, s_{2}\right)$. Then $u$ is continuous on $U$.

(ii) Let $G$ be an open subset of $M \times[T, \infty)$ and $f: G \rightarrow(-\infty, \infty)$ be parabolic for $Y(t)$ on $G$. Then $f$ is continuous on $G$.

3. Semigroups and parabolic functions associated with classical diffusions. In this section, we consider the case of a diffusion process on $R^{d}$ corresponding to continuous coefficients $a=a_{i j}(x, t), 1 \leq i, j \leq d, b=b_{i}(x, t), 1 \leq i \leq d$, and $c=c(x, t) \geq 0$. We assume that for any $T>0$, the matrix $a$ is uniformly positive definite and the coefficients $a_{i j}$ are bounded and Hölder continuous on $R^{d} \times$ $[0, T]$, while the coefficients $b_{i}$ and $c$ are bounded and satisfy a Hölder condition with respect to $x$ on $R^{d} \times[0, T]$. The equation

$$
\frac{1}{2} \sum_{i, j=1}^{d} a_{i j}(x, t) \frac{\partial^{2} u}{\partial x_{i} \frac{\partial x_{j}}{j}}+\sum_{i=1}^{d} b_{i}(x, t) \frac{\partial u}{\partial x_{i}}-c(x, t) u+\frac{\partial u}{\partial t}=0
$$

has a unique fundamental solution $p(s, x ; t, y)$ (see Theorem 0.4 of [5]). The 
function $p(s, x ; t, y)$ defines the transition density of a normal continuous strong Markov process $Y(t)$. It can be shown that the corresponding space-time process $Z(t)$ is a $\hat{C}$ process. The process $Y(t)$ has the strong Feller property; however, $Z(t)$ fails to be a strong Feller process, as it is easy to give examples of bounded measurable functions $f$ such that $\bar{T}_{t} f$ is discontinuous. Because of this, various useful properties of harmonic functions associated with strong Feller processes, which have led to a nice characterization of the class of all harmonic functions for a canonical diffusion (see Chapter 13 of [5]), cannot be applied here.

The (strong) $\hat{C}$-infinitesimal generator of the space-time process $Z(t)$ coincides with the weak $\hat{C}$-infinitesimal generator and is the restriction of the differential operator $L$ to the class

$$
\left\{u \mid u \in \hat{C}, \frac{\partial u}{\partial x_{i}}, \frac{\partial^{2} u}{\partial x_{i} \partial x_{j}}, \frac{\partial u}{\partial t} \in \hat{C}(i, j=1, \ldots, d)\right\},
$$

where $L$ is given by

$$
L u=\frac{\partial u}{\partial t}+\frac{1}{2} \sum_{i, j=1}^{d} a_{i j}(x, t) \frac{\partial^{2} u}{\partial x_{i} \partial x_{j}}+\sum_{i=1}^{d} b_{i}(x, t) \frac{\partial u}{\partial x_{i}}-c(x, t) u .
$$

Now consider the characteristic operator $\mathcal{U}$ of $Z(t)$. Let $G$ be an open subset of $R^{d} \times[0, \infty)$. Let $f: G \rightarrow(-\infty, \infty)$ be continuous and

$$
\frac{\partial f}{\partial t}, \frac{\partial f}{\partial x_{i}}, \frac{\partial^{2} f}{\partial x_{i} \partial x_{j}} \quad(i, j=1, \ldots, d)
$$

be continuous on $G$, and we shall denote the set of all such functions $f$ by $C^{2,1}(G)$. We assert that $f \in \mathfrak{D}_{\mathfrak{U}}(G)$ and $\mathcal{U}_{f}=L f$ on $G$. To prove this, let $\left(x_{0}, s_{0}\right) \in G$ and $U_{0}$ be a compact neighborhood of $\left(x_{0}, s_{0}\right)$. By modifying $f$ outside $U_{0}$, we have a function $g \in C^{2}, 1\left(R^{d} \times[0, \infty)\right)$ such that $g=f$ on $U_{0}$ and $g$ has compact support. Then $g$ is in the domain of the $\hat{C}$-infinitesimal generator and $L g=\mathcal{U}_{g}$. Since $f=g$ on $U_{0}, \mathcal{U}_{f}\left(x_{0}, s_{0}\right)=\mathcal{U}_{g}\left(x_{0}, s_{0}\right)=L g\left(x_{0}, s_{0}\right)$.

Using the estimate $(0.33)$ in [5], for every $T>0$, there exist constants $\alpha_{T}>0, M_{T}>0$ such that

$$
p(s, x ; t, y) \leq M_{T}(t-s)^{-d / 2} \exp \left(-\alpha_{T}|y-x|^{2} /(t-s)\right)
$$

for all $x, y \in R^{d}, 0 \leq s<t \leq T$. Therefore given $\epsilon>0$,

$$
\int_{|x-y| \geq \epsilon} p(s, x ; t, y) d y \leq M_{T} \int_{|z| \geq \epsilon(t-s)}-1 / 2 \exp \left(-\alpha_{T}|z|^{2}\right) d z \rightarrow 0 \text { as } t \downarrow s:
$$

On the other hand, it is easy to see that $P\left(s, x ; s+\delta, R^{d}\right) \uparrow 1$ uniformly for $(x, s)$ in every compact set as $\delta \downarrow 0$. Therefore we have proved that (2) indeed 
holds, and Theorem 2 and its corollary can be applied. Hence if $f$ is parabolic for $Y(t)$ on an open subset $G$ of $R^{d} \times[0, \infty)$, then $f$ is continuous on $G$. In the case where $Y(t)$ is the $d$-dimensional Brownian motion, this result was established by Doob [3]. Actually Doob did not work with the space-time process $Z(t)$, but worked with the Brownian trajectory process $\tilde{Z}(t)=\phi(Z(t))$, where $\phi(x, t)=(x,-t)$. His approach made use of the explicit form of the exit distribution from a rectangular domain $S$ by $\tilde{Z}(t)$, so that if $(x, s)$ is an interior point of $S$, then $E\left[f\left(\widetilde{Z}\left(\tau_{s}\right)\right) \mid Z(0)=(x, s)\right]$ can be expressed in terms of integrals involving the boundary function $f$, the Green's function for the heat equation in $S$ and partial derivatives of the Green's function (cf. [6]), where $\tau_{S}$ denotes the first exit time by $\widetilde{Z}(t)$ from the rectangular domain $S$.

We now assert that the class of all parabolic functions on $G$ for the diffusion $Y(t)$ coincides with the class of all continuous solutions of $\mathcal{U}_{f=0}$ on $G, \mathcal{U}$ being the space-time characteristic operator. Let $V$ be a bounded open subset of $R^{d}$. We say that $a \in \partial V$ (where $\partial V$ denotes the boundary of $V$ ) is a regular point with respect to $Y(t)$ if, for all $s \geq 0, P_{s, a}\left[\sigma_{V}^{s+}>s\right]=0$, where $\sigma_{V}^{s+}=$ $\lim _{\epsilon ! 0} \sigma_{V}^{s+\epsilon}\left(\sigma_{V}^{s+\epsilon}\right.$ being defined as in Theorem 2$)$. Omitting the details here, it can be shown that if $V$ is a bounded open subset of $R^{d}$ such that $\partial V$ is regular with respect to $Y(t)$ and $U=V \times\left[T, T_{1}\right), T_{1}>T \geq 0$, and if $f$ is a bounded continuous function on $R^{d} \times[T, \infty)$, then, for any $t>0, F_{t}(x, s)=E_{s, x} f\left(Z_{T}\left(t \wedge \tau_{U}\right)\right)$ defines a continuous function of $(x, s)$ in $R^{d} \times[T, \infty)$, where $Z_{T}(t)$ is the part of $Z(t)$ on $[T, \infty)$ and $\tau_{U}=\inf \left\{t \geq 0 \mid Z_{T}(t) \notin U\right\}$. The process $Z_{T}\left(t \wedge \tau_{U}\right)$ is therefore a Feller process on $R^{d} \times[T, \infty)$. As in Theorem 13.8 of [5], we can prove that $a \in \partial V$ is a regular point with respect to $Y(t)$ if $\partial V$ is differentiable at $a$, i.e., there exist a neighborhood $K$ of $a$ and a function $\psi(x)$, differentiable at $a$ and having a nonzero differential, such that $K \cap V=\{x \in K \mid \psi(x)<0\}$. Let $G$ be an open subset of $R^{d} \times[T, \infty)$. Then given any open subset $U$ of $R^{d} \times$ $[T, \infty)$ with compact closure contained in $G$, we can find $t_{n}>\cdots>t_{0} \geq T$ $(n \geq 1)$ and disjoint sets $U_{j}^{i}\left(i=1, \ldots, n ; j=1, \ldots, n_{i}\right)$ such that $U_{j}^{i}$ is of the form $V_{j}^{i} \times\left[t_{i-1}, t_{i}\right)$ just considered and the closure of $\widetilde{U}=\bigcup_{i=1}^{n} \bigcup_{j=1}^{n_{i}} U_{j}^{i}$ is contained in $G$, while $\widetilde{U}$ contains $U$. By Theorem 1 , the set of all parabolic functions for $Y(t)$ on $G$ coincides with the set of all continuous solutions of the equation $\mathcal{U} f(x, t)=0,(x, t) \in G$, as we have seen that any parabolic function for $Y(t)$ is continuous.

Let us further assume that, for any $T>0$, the coefficients $b_{i}$ and $c$ also satisfy a Hölder condition with respect to $t$ on $R^{d} \times[0, T]$. Then we assert that the class of all parabolic functions for $Y(t)$ on $G$ coincides with the set of all solutions $f \in C^{2,1}(G)$ of $L f=0$ on $G$, where $L$ is the backward parabolic operator defined by (3). To see this, we shall show that any function $f$ which is 
parabolic for $Y(t)$ on $G$ belongs to $C^{2,1}(G)$. Take any $s_{2}>s_{1} \geq 0$ and any open sphere $V$ in $R^{d}$ such that $\bar{V} \times\left[s_{1}, s_{2}\right] \subset G .(\bar{V}$ denotes the closure of $V$.$) Let$ $D=V \times\left(s_{1}, s_{2}\right), B=V \times\left\{s_{1}\right\}, \Sigma=\left(\partial V \times\left[s_{1}, s_{2}\right)\right) \cup\left(\bar{V} \times\left\{s_{2}\right\}\right)$. Define $\phi: R^{d} \times$ $\left.\underset{\sim}{[0}, s_{2}\right] \rightarrow R^{d} \times\left[0, s_{2}\right]$ by $\phi(x, t)=\left(x, s_{2}-t\right)$, and let $\tilde{a}_{i j}(x, t)=a_{i j}\left(x, s_{2}-t\right)$, $\tilde{b_{i}}(x, t)=b_{i}\left(x, s_{2}-t\right), \tilde{c}(x, t)=\tilde{c}\left(x, s_{2}-t\right)$ and $\tilde{f}(x, t)=f\left(x, s_{2}-t\right)$. Now $\tilde{f}^{-i j}$ is continuous and there exists a unique solution $v$ of the first initial-boundary value problem:

$$
\begin{gathered}
\frac{1}{2} \sum_{i, j=1}^{d} \tilde{a}_{i j}(x, t) \frac{\partial^{2} v}{\partial x_{i} \partial x_{j}}+\sum_{i=1}^{d} \widetilde{b}_{i}(x, t) \frac{\partial v}{\partial x_{i}}-\tilde{c}(x, t) v-\frac{\partial v}{\partial t}=0 \quad \text { on } \phi[D] \cup \phi[B], \\
v=\tilde{f} \text { on } \phi[\Sigma] .
\end{gathered}
$$

Furthermore, $v \in C^{2,1}(\phi[D] \cup \phi[B])$. Let $u(x, t)=v\left(x, s_{2}-t\right),(x, t) \in \bar{D}$. Then $u$ satisfies $L u=0$ on $D \cup B$ and $u \in C^{2,1}(D \cup B)$. Therefore $u \in \mathscr{D}_{\mathfrak{u}}\left(V \times\left[s_{1}, s_{2}\right)\right)$, $\mathcal{U}_{u}=L u=0=\mathcal{U}_{f}$ on $V \times\left[s_{1}, s_{2}\right)$ and $u=f$ on $\Sigma$. This implies that $u=f$ on $\bar{V} \times\left[s_{1}, s_{2}\right]$, in accordance with the following lemma:

Lemma 2. (a) Let $U$ be a bounded open subset of $R^{d} \times\left[s_{1}, s_{2}\right), 0 \leq s_{1}<$ $s_{2}$, of the form $D \cup B_{1}$, where $D$ is an open subset of $R^{d} \times\left(s_{1}, s_{2}\right)$, whose boundary $\partial D$ consists of a domain $B_{1}$ lying on $t=s_{1}$, the closure of a domain $B_{2}$ lying on $t=s_{2}$, and a manifold $S$ lying in the strip $s_{1} \leq t<s_{2}$ such that $S$ bas the outside strong sphere property (see p. 69 of [7]). Then given any $\epsilon>0$, there exists $b: \bar{U} \rightarrow(-\infty, \infty)$ such that $0 \leq b \leq \epsilon, b \in \mathscr{D}_{\mathcal{U}}(U)$ and $\mathcal{U} b<0$ on $U$.

(b) Let $U$ be as in (a). Suppose $f: \bar{U} \rightarrow(-\infty, \infty)$ is continuous, $f \in \mathscr{D}_{\mathcal{U}}(U)$, $\mathcal{U}_{f} \leq 0$ on $U, f \geq 0$ on $\bar{B}_{2} \cup S$. Then $f \geq 0$ on $U$.

Proof. (a) Define $\widetilde{a}_{i j}, \widetilde{b}_{i}, \widetilde{c}, \phi$ as before. Then there exists a unique solution $g$ of the first initial-boundary value problem:

$$
\begin{gathered}
\frac{1}{2} \sum_{i, j} \widetilde{a}_{i j}(x, t) \frac{\partial^{2} g}{\partial x_{i} \partial x_{j}}+\sum_{i} \widetilde{b}_{i}(x, t) \frac{\partial g}{\partial x_{i}}-\widetilde{c}(x, t) g-\frac{\partial g}{\partial t}=-1 \text { on } \phi[D] \cup \phi\left[B_{1}\right], \\
g=0 \text { on } \phi\left[\bar{B}_{2}\right] \cup \phi[S] .
\end{gathered}
$$

Furthermore, $g \in C^{2,1}\left(\phi[D] \cup \phi\left[B_{1}\right]\right)$ and $g$ is continuous on $\phi[\bar{D}]$. It follows from the classical maximum principle that $g \geq 0$ on $\phi[\bar{D}]$. Let $k(x, t)=$ $g\left(x, s_{2}-t\right),(x, t) \in \bar{D}$. Then $k \in C^{2}, 1\left(D \cup B_{1}\right), k$ is continuous on $\bar{D}, k \geq 0$ and $L k=-1$ on $D \cup B_{1}=U$. Therefore $k \in \mathscr{D}_{\mathfrak{U}}(U)$ and $\mathcal{U}_{k}=L k=-1$ on $U$. Given $\epsilon>0$, we can choose a positive constant $c$ such that $c k \leq \epsilon$ on $\bar{U}$. Let $b=c k$. Then $U_{b}=c U_{k}<0$ on $U$.

(b) Given $\epsilon>0$, let $b$ be the function constructed in (a). Let $\tilde{f}=f+b$. 
Then $\mathcal{U} \tilde{f}=\mathcal{U}_{f}+\mathcal{U}_{b}<0$ on $U, \tilde{f} \geq 0$ on $\bar{B}_{2} \cup S$. By the minimum principle for $\mathcal{U}$ (the version we referred to in $\S 2$ ), $\tilde{f} \geq 0$ on $U$. This means that $f \geq-\epsilon$ on $U$. Since $\epsilon$ is arbitrary, the desired conclusion follows. Q.E.D.

Summing up, we have the following theorem.

Theorem 3. Let $Y(t)$ be the diffusion on $R^{d}$ corresponding to diffusion coefficients $a_{i j}(x, t), b_{i}(x, t), i, j=1, \ldots, d$, and $c(x, t) \geq 0$ such that, for each $T>0$, the matrix $a$ is uniformly positive definite and the coefficients $a_{i j}, b_{i}$ and $c$ are bounded and Hölder continuous on $R^{d} \times[0, T]$. If $G$ is an open subset of $R^{d} \times[T, \infty)(T \geq 0)$ and $u: G \rightarrow(-\infty, \infty)$, then the following statements are equivalent:

(A) $u$ is continuous on $G$ and $\mathcal{U}_{u}=0$ on $G$,

(B) $u \in C^{2,1}(G)$ and $L u=0$ on $G$, where $L$ is the differential operator defined in (3),

(C) $u$ is parabolic for $Y(t)$ on $G$,

(D) $u$ is bounded on every compact subset of $G$; and if $U$ is any open subset of $R^{d} \times[T, \infty)$ with compact closure contained in $G$ and $\tau_{U}$ is the first exit time $b v$ the corresponding space-time process $Z(t)$ from $U$, then $u\left(Y\left(s+\left(t \wedge \tau_{U}\right)\right), s+\left(t \wedge \tau_{U}\right)\right), t \geq 0$, is a martingale with respect to $P_{s, x}$ for all $(x, s) \in G$.

4. One-dimensional regular diffusions with inaccessible boundaries. Consider a conservative continuous strong Markov process $X(t)$ on an interval $I$ with endpoints $r_{0}, r_{1}$, where $-\infty \leq r_{0}<r_{1} \leq \infty$, such that all the interior points of $I$ are regular points. Let $C_{I}$ denote the space of all continuous functions on $I$ such that $\lim _{x \rightarrow r_{i}} f(x)$ exists (as a finite number) for $i=0,1$. To simplify the notation, we shall, without loss of generality, assume that $0 \in\left(r_{0}, r_{1}\right), s(0)=m(0)=$ $m\left(0_{-}\right)=0$, where $s$ is the scale (which is unique up to a linear transformation) and the measure which assigns mass $m(b)-m(a)$ to the interval $(a, b]$ for $r_{0}<$ $a<b<r_{1}$ is the speed measure of $X(t)$. Define

$$
u_{1}(x)=\int_{0}^{x} m(z) d s(z), \quad \omega_{1}(x)=\int_{0}^{x} s(z) d m(z) .
$$

$r_{i}$ is called an accessible boundary if $u_{1}\left(r_{i}\right)$ is finite, and is said to be inaccessible if otherwise. An accessible boundary $r_{i}$ is said to be regular if $\omega_{1}\left(r_{i}\right)$ is finite, and is called an exit boundary if otherwise. An inaccessible boundary $r_{i}$ is an entrance boundary if $\omega_{1}\left(r_{i}\right)$ is finite, and is a natural boundary otherwise. It is well known (see Chapter 2 of [9]) that $D_{m} D_{s}^{+}$, when restricted to the class $F_{I}$ of all functions $f \in C_{I}$ such that $D_{m} D_{s}^{+} f \in C_{I}$, is the strong $C_{I}$-infinitesimal generator for the process $X(t)$ if $r_{0}, r_{1}$ are inaccessible boundaries.

Suppose hereafter that $r_{0}, r_{1}$ are inaccessible boundaries. Let $Z(t)$ be the corresponding space-time process and $\mathcal{U}$ the characteristic operator of $Z(t)$. Let $x_{0} \in\left(r_{0}, r_{1}\right)$ and $t_{0} \geq 0$. Let $G$ be an open subset of $\left(r_{0}, r_{1}\right) \times[0, \infty)$ containing 
$\left(x_{0}, t_{0}\right)$ and $f: G \rightarrow(-\infty, \infty)$ such that $\partial f / \partial t$ is continuous at $\left(x_{0}, t_{0}\right)$ and $f\left(x, t_{0}\right), D_{m} D_{s}^{+} f\left(x, t_{0}\right)$ are both continuous in $x$ for all $x$ in some neighborhood of $x_{0}$. Then we assert that $\mathcal{U} f\left(x_{0}, t_{0}\right)$ exists and

$$
\mathcal{U} f\left(x_{0}, t_{0}\right)=D_{m} D_{s}^{+} f\left(x_{0}, t_{0}\right)+\partial f\left(x_{0}, t_{0}\right) / \partial t \text {. }
$$

To prove this, let $U_{\epsilon}$ be any open rectangular neighborhood of $\left(x_{0}, t_{0}\right)$ contained in $G$ such that

$$
\left|\partial f(y, t) / \partial t-\partial f\left(x_{0}, t_{0}\right) / \partial t\right| \leq \epsilon \quad \forall(y, t) \in U_{\epsilon}
$$

Let $U$ be any open neighborhood of $\left(x_{0}, t_{0}\right)$ whose closure is contained in $U_{\epsilon}$, and let $\tau_{U}$ be the first exit time from $U$ by $Z(t)$. Write

$$
\begin{aligned}
E\left[f\left(X\left(t_{0}+\tau_{U}\right), t_{0}+\tau_{U}\right) \mid X\left(t_{0}\right)=x_{0}\right]-f\left(x_{0}, t_{0}\right) & \\
= & E\left[f\left(X\left(t_{0}+\tau_{U}\right), t_{0}+\tau_{U}\right)-f\left(X\left(t_{0}+\tau_{U}\right), t_{0}\right) \mid X\left(t_{0}\right)=x_{0}\right] \\
& +E\left[f\left(X\left(t_{0}+\tau_{U}\right), t_{0}\right)-f\left(x_{0}, t_{0}\right) \mid X\left(t_{0}\right)=x_{0}\right] .
\end{aligned}
$$

Since

$$
f\left(X\left(t_{0}+\tau_{U}\right), t_{0}+\tau_{U}\right)-f\left(X\left(t_{0}+\tau_{U}\right), t_{0}\right)=\tau_{U}(\partial f / \partial t)\left(X\left(t_{0}+\tau_{U}\right), \hat{t}\right),
$$

where $\hat{t}$ lies between $t_{0}$ and $t_{0}+\tau_{U}$, and since $\left(X\left(t_{0}+\tau_{U}\right), \hat{t}\right) \in U_{\epsilon}$, we have

$$
\frac{E\left(\left|f\left(X\left(t_{0}+\tau_{U}\right), t_{0}+\tau_{U}\right)-f\left(X\left(t_{0}+\tau_{U}\right), t_{0}\right)-\tau_{U} \partial f\left(x_{0}, t_{0}\right) / \partial t\right| \mid X\left(t_{0}\right)=x_{0}\right)}{E\left(\tau_{U} \mid X\left(t_{0}\right)=x_{0}\right)} \leq \epsilon .
$$

Let $f_{t_{0}}(x)=f\left(x, t_{0}\right)$. We need only prove that

$$
\lim _{U !\left(x_{0}, t_{0}\right)} \frac{E\left[f_{t_{0}}\left(X\left(t_{0}+\tau_{U}\right)\right)-f_{t_{0}}\left(x_{0}\right) \mid X\left(t_{0}\right)=x_{0}\right]}{E\left(\tau_{U} \mid X\left(t_{0}\right)=x_{0}\right)}=D_{m} D_{s}^{+} f_{t_{0}}\left(x_{0}\right) .
$$

The functions $f_{t_{0}}, D_{m} D_{s}^{+} f_{t_{0}}$ are continuous on $[a, b]$, where $r_{0}<a<x_{0}<b<r_{1}$. Choose $x_{1} \in(a, b)$ such that $m\left(x_{1}\right)=m\left(x_{1}-\right)$. Define

$$
\tilde{f}(x)=f_{t_{0}}(x)-s(x) D_{s}^{+} f_{t_{0}}\left(x_{1}\right), \quad x \in[a, b] .
$$

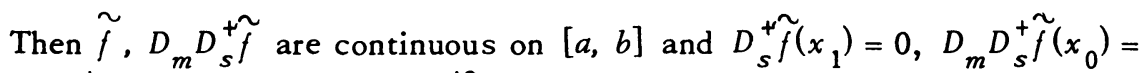
$D_{m} D_{s}^{+} f\left(x_{0}, t_{0}\right)$. Let $b=D_{m} D_{s}^{+} \tilde{f}$ on $[a, b]$. Since $b$ is continuous on $[a, b]$, we can extend $b$ to a continuous function $b_{I}$ on $I$ such that $b_{I}$ vanishes outside $\left[a^{\prime}, b^{\prime}\right]$ with $r_{0}<a^{\prime}<a<b<b^{\prime}<r_{1}$. Define

$$
\hat{f}(x)=\int_{x_{1}}^{x} \int_{x_{1}}^{y} b_{I}(z) d m(z) d s(y)+\widetilde{f}\left(x_{1}\right), \quad x \in I .
$$

Now $\hat{f}$ is a continuous function on $I$, is constant outside $\left[a^{\prime}, b^{\prime}\right]$, and $D_{m} D_{s}^{+} \hat{f}=$ $b_{I}$ is a continuous function on $I$ with compact support. Therefore $\hat{f} \in F_{l}$. Since $D_{m} D_{s}^{+} \tilde{f}$ is continuous on $[a, b]$, we have, for all $x \in[a, b]$,

$$
\tilde{f}(x)=\int_{x_{1}}^{x} \int_{x_{1}}^{y} D_{m} D_{s}^{+\tilde{f}}(z) d m(z) d s(y)+\tilde{f}\left(x_{1}\right)+\left(s(x)-s\left(x_{1}\right)\right) D_{s}^{+\tilde{f}}\left(x_{1}\right) .
$$




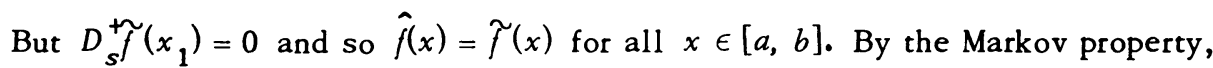
$E\left[\hat{f}\left(X\left(t_{0}+\tau_{U}\right)\right) \mid X\left(t_{0}\right)=x_{0}\right]=E_{x_{0}} \hat{f}\left(X\left(\zeta_{U}\right)\right)$ and $E\left(\tau_{U} \mid X\left(t_{0}\right)=x_{0}\right)=E_{x_{0}} \zeta_{U}$, where $\zeta_{U}=\inf \left\{t \geq 0 \mid\left(X(t), t+t_{0}\right) \in U\right\}$. Since $\hat{f}$ is in the domain $F_{I}$ of the infinitesimal generator, and $\zeta_{U}$ is a Markov time with $E_{x_{0}} \zeta_{U}<\infty$, it follows from Dynkin's formula that

$$
E_{x_{0}} \hat{f}\left(X\left(\zeta_{U}\right)\right)-\hat{f}\left(x_{0}\right)=E_{x_{0}} \int_{0}^{\zeta_{U}} D_{m} D_{s}^{+} \hat{f}(X(s)) d s
$$

Therefore we have

$$
\begin{aligned}
\lim _{U !\left(x_{0}, t_{0}\right)} & \frac{E_{x_{0}} \tilde{f}\left(X\left(\zeta_{U}\right)\right)-\tilde{f}\left(x_{0}\right)}{E_{x_{0}} \zeta_{U}}=\lim _{U !\left(x_{0}, t_{0}\right)} \frac{E_{x_{0}} \hat{f}\left(X\left(\zeta_{U}\right)\right)-\hat{f}\left(x_{0}\right)}{E_{x_{0}} \zeta_{U}} \\
= & \lim _{U !\left(x_{0}, t_{0}\right)}\left(E_{x_{0}} \int_{0}^{\zeta_{U}} D_{m} D_{s}^{+} \hat{f}(X(s)) d s\right) / E_{x_{0}} \zeta_{U} \\
= & D_{m} D_{s}^{+} \hat{f}\left(x_{0}\right)=D_{m} D_{s}^{+} f\left(x_{0}, t_{0}\right) .
\end{aligned}
$$

To complete the proof of (4), it remains to show that

$$
\lim _{U !\left(x_{0}, t_{0}\right)} \frac{E_{x_{0}} s\left(X\left(\zeta_{U}\right)\right)-s\left(x_{0}\right)}{E_{x_{0}} \zeta_{U}}=0
$$

But this is obvious, since $s$ is harmonic for $X(t)$.

Let $r_{0}<a<b<r_{1}$. Define first passage times

$$
\xi_{a}=\inf \{t \geq 0 \mid X(t)=a\}, \quad \xi_{a b}=\xi_{a} \wedge \xi_{b} .
$$

Lemma 3. For $r_{0}<a<x<b<r_{1}, u \geq 0$, define

$$
\begin{gathered}
\Psi_{x}(u)=P_{x}\left[\xi_{a}=\xi_{a b} \leq u\right], \quad \Phi_{x}(u)=P_{x}\left[\xi_{b}=\xi_{a b} \leq u\right], \\
\rho_{1}(x, u)=P_{x}\left[\xi_{b} \leq u\right], \quad \rho_{2}(x, u)=P_{x}\left[\xi_{a} \leq u\right], \quad \rho_{3}(x, u)=P_{x}\left[\xi_{a b} \leq u\right] .
\end{gathered}
$$

Then for $u>0, x_{0} \in(a, b)$ and any continuous function $g:[0, u] \rightarrow(-\infty, \infty)$,

$$
\lim _{x \rightarrow x_{0}} \Psi_{x}(u)=\Psi_{x_{0}}(u), \quad \lim _{x \rightarrow x_{0}} \int_{0}^{u} g(\lambda) d \Psi_{x}(\lambda)=\int_{0}^{u} g(\lambda) d \Psi_{x_{0}}(\lambda)
$$

Similar assertions hold for $\Phi_{x}(u)$. Furthermore, the functions $\rho_{i}(x, u), i=1,2,3$, are jointly continuous in $(x, u)$ for $(x, u) \in(a, b) \times(0, \infty)$. Also $\lim _{x \uparrow b} \rho_{1}(x, u)=$ $\lim _{x ! a} \rho_{2}(x, u)=1$, for all $u>0$.

Proof. We first prove that $\rho_{1}(x, u)$ is jointly continuous in $(x, u)$ for $(x, u) \epsilon$ $\left(r_{0}, b\right) \times(0, \infty)$. Consider the process $\widetilde{X}(t)$ obtained from $X(t)$ by curtailment of its lifetime up to $\xi_{b}$, i.e., $\widetilde{X}(t)$ is the part of $X(t)$ on $\left(r_{0}, b\right)$. Its transition function is determined by $D_{m} D_{s}^{+}$(restricted to $\left(r_{0}, b\right)$ ) subject to the boundary condition $f(b)=0$. This transition function of $\widetilde{X}(t)$ has a density $\widetilde{p}(t, x, y)$ with respect to speed measure and $\tilde{p}$ is continuous on $(0, \infty) \times\left(r_{0}, b\right) \times\left(r_{0}, b\right)$ (see 
p. 149 of [6]). Now

$$
\rho_{1}(x, u)=1-P_{x}\left[\tilde{X}(u) \in\left(r_{0}, b\right)\right]=1-\int_{\left(r_{0}, b\right)} \tilde{p}(u, x, y) m(d y) .
$$

Since $\tilde{p}$ is continuous in $(x, u)$, it follows that $\rho_{1}$ is continuous on $\left(r_{0}, b\right) \times$ $(0, \infty)$. We can prove the assertions for $\rho_{2}, \rho_{3}$ in a similar way.

By making use of the strong Markov property, it is easy to prove that $\lim _{x \rightarrow x_{0}} \Phi_{x}(u)=\Phi_{x_{0}}(u), u>0$. Obviously $\Phi_{x}(0)=0$ for all $x \in(a, b)$. Therefore by the Helly-Bray Lemma, for any continuous function $g$ on $[0, u]$, we have

$$
\lim _{x \rightarrow x_{0}} \int_{0}^{u} g(\lambda) d \Phi_{x}(\lambda)=\int_{0}^{u} g(\lambda) d \Phi_{x_{0}}(\lambda) \text {. }
$$

The assertions for $\Psi_{x}$ can be proved similarly.

To prove that $\lim _{x\rfloor a} \rho_{2}(x, u)=1$ for all $u>0$, we consider the matching number $e_{3}=\lim _{x ! a} E_{x} \exp \left(-\xi_{a}\right)$ (cf. [6]). Since the diffusion is regular, $e_{3}=1$. This implies that, for any $u>0, \rho_{2}(x, u)=P_{x}\left[\xi_{a} \leq u\right] \rightarrow 1$ as $x \downarrow a$. In a similar way, we can prove that $\lim _{x \uparrow b} P_{x}\left[\xi_{b} \leq u\right]=1$. Q.E.D.

Lemma 4. For any compact subset $C$ of $\left(r_{0}, r_{1}\right)$ and any $\epsilon>0$,

$$
\lim _{\delta \backslash 0} \sup _{\substack{t\left(r_{0}, r_{1}\right) \\ t \geq 0}} P_{x}\left[X(t) \in C, \sup _{t \leq t_{1} \leq t \leq t+\delta}\left|X\left(t_{1}\right)-X\left(t_{2}\right)\right|>\epsilon\right]=0 .
$$

Proof. For any open set $U$ whose closure is a compact set in $\left(r_{0}, r_{1}\right)$ and any $\epsilon>0$,

$$
\lim _{t \downarrow 0} \sup _{x \in U} t^{-1} P\left(t, x,\left(r_{0}, r_{1}\right)-(x-\epsilon, x+\epsilon)\right)=0
$$

(cf. [5]). Therefore we can apply Lemma 13.2 of [5] and obtain that, for any $\epsilon>0$ and any open set $U$ whose closure is a compact subset of $\left(r_{0}, r_{1}\right)$,

$$
\lim _{u \in 0} \sup _{x \in U} P_{x}\left[\sup _{0 \leq t \leq t_{2} \leq u}\left|X\left(t_{1}\right)-X\left(t_{2}\right)\right|>\epsilon\right]=0 .
$$

Let $C$ be a compact subset of $\left(r_{0}, r_{1}\right)$ and $\epsilon>0$. Given $\eta>0$, choose $\delta_{0}>0$ such that

$$
\sup _{y \in C} P_{y}\left[\sup _{0 \leq t_{1} \leq t_{2} \leq \delta_{0}}\left|X\left(t_{1}\right)-X\left(t_{2}\right)\right|>\epsilon\right]<\eta .
$$

Then for $x \in\left(r_{0}, r_{1}\right), t \geq 0, \delta<\delta_{0}$,

$$
\begin{aligned}
P_{x} & {\left[X(t) \in C, \sup _{t \leq t_{1} \leq t_{2} \leq t+\delta}\left|X\left(t_{1}\right)-X\left(t_{2}\right)\right|>\epsilon\right] } \\
& =\int_{[X(t) \in C]} P_{X(t)}\left[\sup _{0 \leq t_{1} \leq t_{2} \leq \delta}\left|X\left(t_{1}\right)-X\left(t_{2}\right)\right|>\epsilon\right] d P_{x} \leq \eta . \quad \text { Q.E.D. }
\end{aligned}
$$

Lemma 5. Let $r_{0}<a<b<r_{1}, T>T_{1} \geq 0$ and $U=(a, b) \times\left[T_{1}, T\right)$. Let $Z(t)$ be the space-time process of $X(t)$, and $Z_{1}(t)$ be the part of $Z(t)$ on $\left(r_{0}, r_{1}\right) \times$ $\left[T_{1}, \infty\right)$. Let $\tau_{U}$ denote the first exit time of $U$ by $Z_{1}(t)$. Then the process $Z_{1}\left(t \wedge \tau_{U}\right), t \geq 0$, is a Feller process on $\left(r_{0}, r_{1}\right) \times\left[T_{1}, \infty\right)$. 
Proof. Let $f$ be any continuous function on $\left(r_{0}, r_{1}\right) \times\left[T_{1}, \infty\right)$. We shall prove that, given $t>0$, the function

$$
F_{t}(x, s)=E\left[f\left(Z_{1}\left(t \wedge \tau_{U}\right)\right) \mid Z_{1}(0)=(x, s)\right]
$$

is a continuous function on $\left(r_{0}, r_{1}\right) \times\left[T_{1}, \infty\right)$. Let $\xi_{a}, \xi_{b}, \xi_{a b}$ be the first passage times defined by (5) and write $\xi=\xi_{a b}$. In the case $t+s \leq T$, we have

$$
\begin{aligned}
F_{t}(x, s) & =\int_{[\xi>t]} f(X(t), t+s) d P_{x}+\int_{\left[\xi=\xi_{a \leq t]}\right.} f(a, \xi+s) d P_{x}+\int_{\left[\xi=\xi_{b \leq t}\right]} f(b, \xi+s) d P_{x} \\
& =\pi_{1}(x, s)+\nu_{1}(x, s)+\mu_{1}(x, s), \text { say. }
\end{aligned}
$$

On the other hand, if $t+s \geq T$, then

$$
\begin{aligned}
F_{t}(x, s)= & \int_{[\xi>T-s]} f(X(T-s), T) d P_{x}+\int_{\left[\xi=\xi_{a} \leq T-s\right]} f(a, \xi+s) d P_{x} \\
& +\int_{\left[\xi=\xi_{b} \leq T-s\right]} f(b, \xi+s) d P_{x} \\
= & \pi_{2}(x, s)+\nu_{2}(x, s)+\mu_{2}(x, s), \text { say. }
\end{aligned}
$$

Making use of Lemmas 3 and 4 , it is not hard to show that $\pi_{i}, \nu_{i}$ and $\mu_{i}(i=1,2)$ are continuous on $U$. Hence $F_{t}$ is continuous on $U$.

We now prove the continuity of $F_{t}$ on the boundary of $U$ (in the space $\left.\left(r_{0}, r_{1}\right) \times\left[T_{1}, \infty\right)\right)$. We first show that

$$
\lim _{\substack{s \mid T \\ x \rightarrow x_{1}, x \in(a, b)}} F_{t}(x, s)=f\left(x_{1}, T\right) \text { for any } x_{1} \in[a, b] .
$$

For $s$ sufficiently close to $T, t+s>T$ and so

$$
\begin{aligned}
F_{t}(x, s) & =\int f(X(\xi \wedge(T-s)),(\xi+s) \wedge T) d P_{x} \\
= & f(x, T)+\int_{[|X(\xi \wedge(T-s))-x| \leq \delta]}\{f(X(\xi \wedge(T-s)),(\xi+s) \wedge T)-f(x, T)\} d P_{x} \\
& +\int_{\left[\left|X\left(\xi \wedge^{\prime}(T-s)\right)-x\right|>\delta\right]}\{f(X(\xi \wedge(T-s)),(\xi+s) \wedge T)-f(x, T)\} d P_{x},
\end{aligned}
$$

where $\delta$ is chosen as follows. Given $\epsilon>0$, we choose $\delta \epsilon(0, t)$ such that $|f(y, s)-f(z, T)|<\epsilon$ if $T-\delta \leq s \leq T$ and $|y-z| \leq \delta, z \in[a, b]$. Then for $x \epsilon$ $(a, b)$ and $s \in[T-\delta, T]$,

$$
\int_{[|X(\xi \wedge(T-s))-x| \leq \delta]}|f(X(\xi \wedge(T-s)),(\xi+s) \wedge T)-f(x, T)| d P_{x}<\epsilon .
$$

By Lemma 4, we can choose $\delta_{1} \epsilon(0, \delta)$ such that

$$
P_{x}\left[\sup _{0 \leq t_{1} \leq t_{2} \leq \delta_{1}}\left|X\left(t_{1}\right)-X\left(t_{2}\right)\right|>\delta\right]<\epsilon \text { for all } x \in(a, b) \text {. }
$$

Therefore if $x \in(a, b)$ and $s \in\left[T-\delta_{1}, T\right]$, then

$$
\int_{[|X(\xi \wedge(T-s))-x|>\delta]}|f(X(\xi \wedge(T-s)),(\xi+s) \wedge T)-f(x, T)| d P_{x} \leq 2\|f\| \epsilon .
$$

Since $\lim _{x \rightarrow x_{1}} f(x, T)=f\left(x_{1}, T\right)$, (6) follows. Finally, making use of Lemma 3, it is easy to show that if $T, \leq s_{0}<T$, then 


$$
\lim _{\substack{s \rightarrow s_{0} \\ x \backslash a}} F_{t}(x, s)=f\left(a, s_{0}\right) \text { and } \lim _{\substack{s \rightarrow s_{0} \\ x \uparrow b}} F_{t}(x, s)=f\left(b, s_{0}\right) . \quad \text { Q.E.D. }
$$

Let $G$ be an open subset of $\left(r_{0}, r_{1}\right) \times[T, \infty) \quad(T \geq 0)$. Then given any open subset $U$ of $\left(r_{0}, r_{1}\right) \times[T, \infty)$ with compact closure contained in $G$, we can find $t_{n}$ $>\ldots>t_{0} \geq T$ and disjoint sets $U_{j}^{i}\left(i=1, \ldots, n ; j=1, \ldots, n_{i}\right)$ such that $U_{j}^{i^{n}}$ is of the form $\left(a_{j}^{i}, b_{j}^{i}\right) \times\left[t_{i-1}, t_{i}\right), r_{0}<a_{j}^{i}<b_{j}^{i}<r_{1}$, and the closure of $\widetilde{U}$ is contained in $G$, while $\widetilde{U}$ contains $U$, where $\widetilde{U}=\bigcup_{i=1}^{n} \bigcup_{j=1}^{n_{i}} U_{j}^{i}$. Let $Z_{i}(t)$ be the part of the space-time process $Z(t)$ on $\left(r_{0}, r_{1}\right) \times\left[t_{i-1}, \infty\right)$ and let $\tau_{i j}=\inf \left\{t \geq 0 \mid Z_{i}(t) \notin U_{j}^{i}\right\}$. By Lemma $5, Z_{i}\left(t \wedge r_{i j}\right)$ is a Feller process. Hence Theorem 1 can be applied and the set of all continuous parabolic functions for $X(t)$ on $G$ coincides with the set of all continuous solutions of the equation $\mathcal{U} f(x, t)=0,(x, t) \in G$, where $\mathcal{U}$ is the space-time characteristic operator. In particular, if $f: G \rightarrow(-\infty, \infty)$ is continuous, $\partial f / \partial t$ is continuous and $D_{m} D_{s}^{+} f$ exists and satisfies $\partial f / \partial t+D_{m} D_{s}^{+} f=0$ on $G$, then $f(X(t \wedge \tau), s+(t \wedge \tau)), t \geq 0$, is a martingale with respect to $P_{x}$ for any $(x, s) \in G$, where $\tau=\inf \{t \geq 0 \mid(X(t), t+s) \notin U\}$ and $U$ is an arbitrary open subset of $\left(r_{0}, r_{1}\right) \times[T, \infty)$ with compact closure contained in $G$. Furthermore, it can be shown that Theorem 2 is applicable here, and so if $f$ is parabolic for $X(t)$ on $G$, then it has to be continuous on $G$.

Let us now consider solutions of $\partial f / \partial t+D_{m} D_{s}^{+} f=0$ obtained by the separation of variables, viz., $f(x, t)=g(x) b(t)$. Such solutions have the form $f(x, t)=$ $e^{-a t} g(x)$, where $\alpha$ is a real number and $D_{m} D_{s}^{+} g(x)=\alpha g(x)$. As before, we assume for notational convenience that $s(0)=m(0)=m(0-)=0$. A solution of $D_{m} D_{s}^{+} g=$ $\alpha g$ is given by

$$
u(x, \alpha)=\sum_{n=0}^{\infty} \alpha^{n} u_{n}(x), \quad x \in\left(r_{0}, r_{1}\right)
$$

where

$$
u_{0}(x)=1, \quad u_{n+1}(x)=\int_{0}^{x} \int_{0}^{y} u_{n}(z) d m(z) d s(y) .
$$

The functions $u_{j}(x), j \geq 1$, are everywhere nonnegative, increase for $x>0$ and decrease for $x<0$, and since $u_{k}(x) \leq u_{j}(x)\left(u_{1}(x)\right)^{k-j} /(k-j)$ ! for $j \leq k$, the series $\sum_{n=0}^{\infty} \alpha^{n} u_{n}(x)$ converges for all real $\alpha$. Another solution of $D_{m} D_{s}^{+} g=a g$ is given by

$$
v(x, \alpha)=\sum_{n=0}^{\infty} \alpha^{n} v_{n}(x), \quad x \in\left(r_{0}, r_{1}\right)
$$

where

$$
v_{0}(x)=s(x), \quad v_{n+1}(x)=\int_{0}^{x} \int_{0}^{y} v_{n}(z) d m(z) d s(y) .
$$

The functions $v_{n}(x)$ are increasing on $\left(r_{0}, r_{1}\right)$, negative if $x<0$, and positive if $x>0$. For $n=0,1,2, \ldots$, 


$$
\begin{gathered}
v_{n}(x) \leq \frac{s(x)}{n !}\left(\int_{0}^{x} s(y) d m(y)\right)^{n}, \quad x>0, \\
\left|v_{n}(x)\right| \leq \frac{|s(x)|}{n !}\left(\int_{x}^{0}|s(y)| d m(y)\right)^{n}, \quad x<0 .
\end{gathered}
$$

Therefore the series $\sum_{n=0}^{\infty} \alpha^{n} v_{n}(x)$ converges for all real $a$.

Consider the solutions of

$$
D_{m} D_{s}^{+} g=\lambda g \quad(\lambda>0)
$$

We have seen that $u(x, \lambda), v(x, \lambda)$ are solutions of (9). Since $\lambda>0$, we have $1+$ $\lambda u_{1}(x) \leq u(x, \lambda) \leq \exp \left(\lambda u_{1}(x)\right)$, and the functions

$$
\begin{aligned}
& u_{+}(x, \lambda)=u(x, \lambda) \int_{x}^{r_{1}} \frac{d s(y)}{(u(y, \lambda))^{2}}, \\
& u_{-}(x, \lambda)=u(x, \lambda) \int_{r_{0}}^{x} \frac{d s(y)}{(u(y, \lambda))^{2}},
\end{aligned}
$$

are respectively positive decreasing and positive increasing solutions of (9) (cf. [9]). As both boundaries are inaccessible, $D_{s}^{+} u_{+}\left(r_{1}, \lambda\right)=D_{s}^{+} u_{-}\left(r_{0}, \lambda\right)=0$. If $r_{1}$ is not an entrance boundary, then $u_{+}\left(r_{1}, \lambda\right)=0$, and if $r_{0}$ is not an entrance boundary, then $u_{-}\left(r_{0}, \lambda\right)=0$. The solutions $u(x, \lambda), v(x, \lambda), u_{+}(x, \lambda), u_{-}(x, \lambda)$ of (9) are all continuous functions in $x$. By our previous result, any continuous solution of $D_{m} D_{s}^{+} f+\partial f / \partial t=0$ with $\partial f / \partial t$ continuous on $\left(r_{0}, r_{1}\right) \times[0, \infty)$ is parabolic for $X(t)$. In particular, $e^{-\lambda t} u(x, \lambda), e^{-\lambda t} v(x, \lambda), e^{-\lambda t} u_{+}(x, \lambda)$ and $e^{-\lambda t} u_{-}(x, \lambda)$ are parabolic for $X(t)$, and so if $V_{n}$ is an interval such that $\bar{V}_{n} \subset\left(r_{0}, r_{1}\right)$ and $\sigma_{n}$ denotes the first exit time from $V_{n}$ by $X(t)$, then $\left\{\exp \left(-\lambda\left(t \wedge \sigma_{n}\right)\right) u\left(X\left(t \wedge \sigma_{n}\right), \lambda\right)\right.$, $t \geq 0\}$, etc., are martingales with respect to $P_{x}$ for all $x \in\left(r_{0}, r_{1}\right)$. Now suppose that $V_{n} \uparrow\left(r_{0}, r_{1}\right)$ as $n \uparrow \infty$. Then $\sigma_{n} \uparrow \infty$ since the boundaries are inaccessible, and it is natural to ask if $\left\{e^{-\lambda t} u(X(t), \lambda), t \geq 0\right\}$, etc., are still martingales. We shall show below that if both boundaries are natural, then the answer to the above question is affirmative.

Theorem 4. Let $X(t)$ be a conservative regular continuous strong Markov process on an interval I with endpoints $r_{0}, r_{1}$, where $-\infty \leq r_{0}<r_{1} \leq \infty$. Let $f$ : $I \times[0, \infty) \rightarrow(-\infty, \infty)$. For $r_{0}<a<b<r_{1}$, let $\xi_{a}, \xi_{b}, \xi_{a b}$ be the first passage times defined by (5). Let $x \in\left(r_{0}, r_{1}\right)$. In order that $f(X(t), t+r), t \geq 0$, is a martingale with respect to $P_{x}$ for all $r \geq 0$, it is necessary that the following conditions bold for all $t \geq 0, r \geq 0$,

$$
\lim _{a ! r_{0}} \int_{\left[\xi_{a<r]}\right.} f\left(a, t+\xi_{a}\right) d P=0 \text { if } r_{0} \text { is inaccessible, }
$$

$$
\lim _{b \mid r} \int_{\left[\xi_{b}<r\right]} f\left(b, t+\xi_{b}\right) d P_{x}=0 \quad \text { if } r_{1} \text { is inaccessible, }
$$




$$
\lim _{\substack{a \downarrow_{0} \\ b r_{1}}} \int_{\left[\xi_{a b} \geq r\right]} f(X(r), t+r) d P_{x}=\int f(y, t+r) P(r, x, d y)
$$

if botb $r_{0}, r_{1}$ are inaccessible,

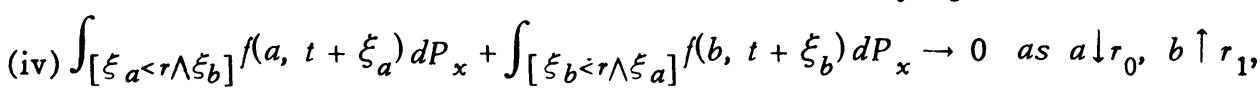
if botb $r_{0}, r_{1}$ are inaccessible.

Conversely, if $r_{0}, r_{1}$ are both inaccessible boundaries, $f$ is parabolic for $X(t)$, and conditions (iii), (iv) bold for all $x \in\left(r_{0}, r_{1}\right)$ and all $t \geq 0, r \geq 0$, then $f(X(t), t+r), t \geq 0$, is a martingale with respect to $P_{x}$ for all $x \in\left(r_{0}, r_{1}\right)$ and all $r \geq 0$.

Proof. Suppose $f(X(t), t+r), t \geq 0$, is a martingale with respect to $P_{x}$ for all $r \geq 0$. Let $r_{0}$ be inaccessible. For any $a \in\left(r_{0}, r_{1}\right), f\left(X\left(t \wedge \xi_{a}\right),\left(t \wedge \xi_{a}\right)+r\right)$, $t \geq 0$, is also a matringale with respect to $P_{x}$ for all $r \geq 0$. Therefore for any $r \geq$ $0, t \geq 0$,

$$
f(x, t)=\int_{\left[\xi_{a \geq r]}\right.} f(X(r), r+t) d P_{x}+\int_{\left[\xi_{a}<r\right]} f\left(a, \xi_{a}+t\right) d P_{x} .
$$

Since $r_{0}$ is inaccessible, $P_{x}\left[\lim _{a l_{0}} \xi_{a}=\infty\right]=1$. Also $E_{x} f(X(r), t+r)=f(x, t)$. Therefore as $a \downarrow r_{0}$,

$$
\int_{\left[\xi_{a \geq r}\right]} f(X(r), t+r) d P_{x} \rightarrow \int f(X(r), t+r) d P_{x}=f(x, t)
$$

and so (i) holds. In a similar way, we prove (ii).

Now assume that both $r_{0}, r_{1}$ are inaccessible. For $r_{0}<a<b<r_{1}$, $f\left(X\left(t \wedge \xi_{a b}\right),\left(t \wedge \xi_{a b}\right)+r\right), t \geq 0$, is a martingale with respect to $P_{x}$ for all $r \geq 0$. Therefore for any $r \geq 0, t \geq 0$,

$$
\begin{aligned}
f(x, t)= & \int_{\left[\xi_{a b \geq r]}\right.} f(X(r), r+t) d P_{x} \\
& +\int_{\left[\xi_{a}<\xi_{b}, \xi_{\left.a^{<r}\right]}\right.} f\left(a, \xi_{a}+t\right) d P_{x}+\int_{\left[\xi_{b}<\xi_{a}, \xi_{b^{<}}\right]} f\left(b, \xi_{b}+t\right) d P_{x^{*}}
\end{aligned}
$$

Since $r_{0}, r_{1}$ are both inaccessible, $P_{x}\left[\lim _{\left.a\right|_{0} ; b \mid r}, \xi_{a b}=\infty\right]=1$. Therefore

$$
\lim _{\substack{a r_{0} \\ b r_{1}}} \int_{\left[\xi_{a b} \geq r\right]} f(X(r), t+r) d P_{x}=\int f(X(r), t+r) d P_{x}=f(x, t)
$$

and so

$$
\lim _{\substack{\left.a\right|_{0} \\ b i_{1}}}\left\{\int_{\left[\xi_{a}<r \wedge \xi_{b}\right]} f\left(a, \xi_{a}+t\right) d P_{x}+\int_{\left[\xi_{b}<r \wedge \xi_{a}\right]} f\left(b, \xi_{b}+t\right) d P_{x}\right\}=0 .
$$

Conversely, suppose that $r_{0}, r_{1}$ are both inaccessible, $f$ is parabolic for $X(t)$ and conditions (iii), (iv) both hold. Since $f$ is parabolic, for any $r_{0}<a<b$ 
$<r_{1}, f\left(X\left(t \wedge \xi_{a b}\right),\left(t \wedge \xi_{a b}\right)+r\right), t \geq 0$, is a martingale with respect to $P_{x}$ for all $x \in\left(r_{0}, r_{1}\right)$ and $r \geq 0$. Therefore for any $x \in\left(r_{0}, r_{1}\right), t \geq 0, r \geq 0,(11)$ holds, and letting $a \downarrow_{r_{0}}, b \uparrow r_{1}$ in (11), we obtain by (iii) and (iv) that $f(x, t)=$ $\int f(y, t+r) P(r, x, d y)$. Hence $f(X(t), t+r), t \geq 0$, is a martingale with respect to $P_{x}$ for all $x \in\left(r_{0}, r_{1}\right)$ and $s \geq 0$. Q.E.D.

Lemma 6. Assume that $r_{0}, r_{1}$ are natural boundaries. If $g(x)$ is any solution of (9), then for any $t>0$ and any $x \in\left(r_{0}, r_{1}\right)$,

(i) $\int|g(y)| P(t, x, d y)<\infty$, and $\int_{[\xi a b \geq t]} g(X(t)) d P_{x} \rightarrow \int g(y) P(t, x, d y)$ as $a \downarrow$ $r_{0}$ and $b \uparrow r_{1}$,

(ii) $\lim _{a ! r} g(a) P_{x}\left[\xi_{a}<t\right]=0$,

(iii) $\lim _{\left.b\right|_{1} g} g(b) P_{x}\left[\xi_{b}<t\right]=0$.

Proof. Since $g$ is a solution of (9), there exist constants $c_{1}, c_{2}$ such that $g(x)=c_{1} u_{+}(x, \lambda)+c_{2} u_{-}(x, \lambda)$. Therefore we need only prove (i), (ii) and (iii) for $u_{+}$and $u$. We first consider $u$. Since $u>0$ on $\left(r_{0}, r_{1}\right)$, the monotone convergence theorem implies that

$$
\lim _{a\left\lfloor r_{0} ; b \dagger r_{1}\right.} \int_{[\xi a b \geq t]} u_{-}(X(t), \lambda) d P_{x}=\int u_{-}(X(t), \lambda) d P_{x} .
$$

Now $u_{-}\left(X\left(t \wedge \xi_{a b}\right), \lambda\right) \exp \left(-\lambda\left(t \wedge \xi_{a b}\right)\right), t \geq 0$, is a martingale with respect to $P_{x}$ and so

$$
u_{-}(x, \lambda) \geq e^{-\lambda t} \int_{\left[\xi_{a b} \geq t\right]} u_{-}(X(t), \lambda) d P_{x} .
$$

Therefore $\int u_{-}(y, \lambda) P(t, x, d y)<\infty$ and (i) is proved. Since $u_{-}\left(r_{0}, \lambda\right)=0$, (ii) is

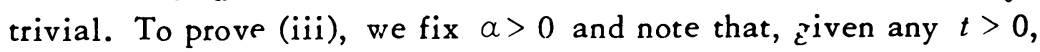

$$
\begin{aligned}
u_{-}(b, \lambda) P_{x}\left[\xi_{b}<t\right] & \leq e^{(a+\lambda) t} u_{-}(b, \lambda) \int_{0}^{\infty} e^{-(a+\lambda) \tau} d P_{x}\left[\xi_{b}<\tau\right] \\
& =e^{(a+\lambda) t} u_{-}(b, \lambda) u_{-}(x, a+\lambda) / u_{-}(b, \alpha+\lambda), \text { if } b>x .
\end{aligned}
$$

Therefore we need only prove that $\lim _{b \mid r_{1}} u_{-}(b, \lambda) / u_{-}(b, a+\lambda)=0$. To prove this, it suffices to show that $\lim _{b r_{1}} u(b, \lambda) / u(b, a+\lambda)=0$. Given any $\delta>0$, we can choose $n_{0}$ such that $\lambda^{n} /(\lambda+\alpha)^{n}<\delta$ for all $n \geq n_{0}$. Then

$$
\frac{u(b, \lambda)}{u(b, \alpha+\lambda)} \leq \frac{\sum_{n=0}^{n_{0}} \lambda^{n} u_{n}(b)}{\sum_{n=n_{0}+1}^{\infty}(\alpha+\lambda)^{n} u_{n}(b)}+\delta \rightarrow \delta \quad \text { as } b \uparrow_{r_{1}} .
$$

The last relation above holds by Lemma 7 below. Since $\delta$ is arbitrary, we indeed have $\lim _{b i r_{1}} u(b, \lambda) / u(b, a+\lambda)=0$. In a similar way, we can prove that (i), (ii), (iii) hold for $u_{+}$, Q.E.D.

Lemma 7. Assume that $r_{1}$ is a natural boundary.

(i) $\int_{0}^{r} 1 u_{n}(x) d m(x)=\infty$ for all $n \geq 1$.

(ii) For $n=0,1,2, \ldots, \lim _{b \mid r} u_{n+1}(b)=\infty, \lim _{b \mid r 1} u_{n+1}(b) / u_{n}(b)=\infty$. 
(iii) For $n=0,1,2, \cdots$ and $\lambda>0, \lim _{\left.b\right|_{1}} u_{n}(b) / u(b, \lambda)=0$.

Similar statements bold for $r_{0}$ if it is natural.

Proof. To prove (i), let $n \geq 1$ and $r_{0}<0<b<r_{1}$. Then since $u_{n-1}$ is continuous and positive on $\left(0, r_{1}\right)$ and $m$ is strictly increasing, it follows that $\int_{0}^{b} u_{n-1}(z) d m(z)>0$. Letting $C=\int_{0}^{b} u_{n-1}(z) d m(z)$, we have

$$
\begin{aligned}
\int_{0}^{r_{1}} u_{n}(x) d m(x) & \geq \int_{b}^{r_{1}} \int_{b}^{x} \int_{0}^{y} u_{n-1}(z) d m(z) d s(y) d m(x) \\
& \geq C \int_{b}^{r_{1}}(s(x)-s(b)) d m(x)=\infty .
\end{aligned}
$$

We shall prove (ii) by induction. Obviously (ii) is true for $n=0$ and it is easy to show that (ii) also holds for $n=1$. Now assume that (ii) holds for $n=k-1$ $(k \geq 2)$. By induction assumption, $\lim _{b \varphi_{r}} u_{k}(b) / u_{k-1}(b)=\infty$. Hence given $\Delta>0$, we can choose $b_{0} \in\left(0, r_{1}\right)$ such that $u_{k}(b) / u_{k-1}(b)>\Delta$ if $b \in\left[b_{0}, r_{1}\right)$. Then for $b \in\left(b_{0}, r_{1}\right)$,

$$
\begin{aligned}
\frac{u_{k+1}(b)}{u_{k}(b)} & \geq \frac{\Delta \int_{b_{0}}^{b} \int_{b_{0}}^{y} u_{k-1}(z) d m(z) d s(y)}{\int_{0}^{b} \int_{0}^{y} u_{k-1}(z) d m(z) d s(y)} \\
& =\Delta\left[\frac{\int_{0}^{b} \int_{0}^{y} u_{k-1}(z) d m(z) d s(y)}{\int_{b_{0}}^{b} \int_{b_{0}}^{y} u_{k-1}(z) d m(z) d s(y)}+\frac{\int_{b_{0}}^{b} \int_{0}^{b_{0}} u_{k-1}(z) d m(z) d s(y)}{\int_{b_{0}}^{b} \int_{b_{0}}^{y} u_{k-1}(z) d m(z) d s(y)}+1\right]^{-1} \\
& =\Delta\left[G_{1}(b)+G_{2}(b)+1\right]^{-1}, \text { say }
\end{aligned}
$$

It is easy to see that $\int_{b_{0}}^{r_{1}} \int_{b_{0}}^{y} u_{k-1}(z) d m(z) d s(y)=\infty$, and therefore we have $\lim _{b{ }_{1} 1} G_{1}(b)=0$. If $s\left(r_{1}\right)<\infty$, then

$$
\int_{b_{0}}^{r_{1}} \int_{0}^{b_{0}} u_{k-1}(z) d m(z) d s(y)<\infty
$$

and so $\lim _{\left.b\right|_{1} \mathfrak{l}} G_{2}(b)=0$. Suppose $s\left(r_{1}\right)=\infty$. By (i), given any $\delta>0$, we can choose $b_{1} \in\left(b_{0}, r_{1}\right)$ such that

$$
\int_{b_{0}}^{b_{1}} u_{k-1}(x) d m(x)>\delta^{-1}
$$

Then for $b \in\left(b_{1}, r_{1}\right)$,

$$
\begin{gathered}
G_{2}(b) \leq \delta u_{k-1}\left(b_{0}\right) m\left(b_{0}\right)\left(s(b)-s\left(b_{0}\right)\right) /\left(s(b)-s\left(b_{1}\right)\right) \\
\rightarrow \delta u_{k-1}\left(b_{0}\right) m\left(b_{0}\right) \quad \text { as } b \uparrow r_{1} .
\end{gathered}
$$

Since $\delta$ is aribtrary, $\lim _{b \mid r_{1}} G_{2}(b)=0$. Therefore (12) implies that $\lim \inf _{b\left\lceil r_{1}\right.} u_{k+1}(b) / u_{k}(b) \geq \Delta$. But $\Delta$ is arbitrary, and so $\lim _{b\left\lceil r_{1}\right.} u_{k+1}(b) / u_{k}(b)=$ $\infty$. Hence we have proved (ii), and (iii) is an obvious consequence of (ii). Q.E.D.

In a similar way, we can prove the following lemma. 
Lemma 8. Assume that $r_{1}$ is a natural boundary.

(i) $\int_{0}^{r} 1 v_{n}(x) d m(x)=\infty$ for all $n \geq 0$.

(ii) For $n=0,1,2, \cdots, \lim _{b ! r} v_{n+1}(b)=\infty, \lim _{b \mid r} v_{n+1}(b) / v_{n}(b)=\infty$.

(iii) For $n=0,1,2, \ldots$ and $\lambda>0, \lim _{b \mid r_{1}} v_{n}(b) / v(b, \lambda)=0$.

Similar statements bold for $r_{0}$ if it is natural.

Theorem 5. Let $X(t)$ be a conservative regular continuous strong Markov process on $\left(r_{0}, r_{1}\right)$ such that the boundaries $r_{0}, r_{1}$ are natural. Let $s(x)$ be the scale and $m$ the speed measure of $X(t)$.

(i) If $f:\left(r_{0}, r_{1}\right) \times[0, \infty) \rightarrow(-\infty, \infty)$ is continuous, $\partial f / \partial t$ is also continuous and $D_{m} D_{s}^{+} f$ exists and satisfies $\partial f / \partial t+D_{m} D_{s}^{+} f=0$ on $\left(r_{0}, r_{1}\right) \times[0, \infty)$, then $f$ is parabolic for $X(t)$. If moreover, for any $T>0$, there exist $\alpha_{T}>0$ and a function $g_{T}:\left(r_{0}, r_{1}\right) \rightarrow(-\infty, \infty)$ satisfying $D_{m} D_{s}^{+} g_{T}=a_{T} g_{T}$ sucb that $\max _{0 \leq t \leq T}|f(x, t)|=$ $O\left(\left|g_{T}(x)\right|\right)$ as $x \uparrow r_{1}$ and as $x \downarrow r_{0}$, then $f(X(t), t+r), t \geq 0$, is a martingale with respect to $P_{x}$ for all $x \in\left(r_{0}, r_{1}\right)$ and all $r \geq 0$.

(ii) For any real number $a$,

$$
\begin{aligned}
& D_{m} D_{s}^{+} g(x)=a g(x) \text { for all } x \in\left(r_{0}, r_{1}\right) \\
& \quad \Rightarrow e^{-\alpha t} g(X(t)), t \geq 0 \text {, is a martingale with respect to } P_{x} \text { for all } x \in\left(r_{0}, r_{1}\right) .
\end{aligned}
$$$$
D_{m} D_{s}^{+} g(x)=\alpha \text { for all } x \in\left(r_{0}, r_{1}\right)
$$$$
\Rightarrow g(X(t))-\alpha t, t \geq 0 \text {, is a martingale with respect to } P_{x} \text { for all } x \in\left(r_{0}, r_{1}\right) \text {. }
$$

(iii) If $f(x, t)=t^{n} \phi_{0}(x)+t^{n-1} \phi_{1}(x)+\cdots+\phi_{n}(x)$ satzsfies $D_{m} D_{s}^{+} f+$ $\partial f / \partial t=0$, then $f(X(t), t+r), t \geq 0$, is a martingale with respect to $P_{x}$ for all $x \in$ $\left(r_{0}, r_{1}\right)$ and all $r \geq 0$.

Proof. We may without loss of generality assume that $0 \in\left(r_{0}, r_{1}\right)$ and $s(0)=$ $m(0)=m(0-)=0$.

To prove (i), we can use Lemma 6 and the assumption that $\max _{0 \leq t \leq T}|f(x, t)|$ $=O\left(\left|g_{T}(x)\right|\right)$ to show that the function $f$ satisfies conditions (iii) and (iv) of Theorem 4. Hence by that theorem, $f(X(t), t+r), t \geq 0$, is a martingale with respect to $P_{x}$ for $x \in\left(r_{0}, r_{1}\right)$ and $r \geq 0$.

To prove (ii), first consider solutions of $D_{m} D_{s}^{+} g=\alpha$. For all $x \in\left(r_{0}, r_{1}\right)$, we have

$$
g(x)=\alpha u_{1}(x)+g(0)+v_{0}(x) D_{s}^{+} g(0) .
$$

Set $f(x, t)=g(x)-a t$. Then $D_{m} D_{s}^{+} f+\partial f / \partial t=0$, and $f$ is continuous. Take $\lambda>$ 0 . By Lemmas 7 and 8 , there exist constants $K_{1}, K_{2}$ such that $u_{1}(x) \leq K_{1} u(x, \lambda)$ and $\left|v_{0}(x)\right| \leq K_{2}|v(x, \lambda)|$ for all $x \in\left(r_{0}, r_{1}\right)$. The desired conclusion then follows from (i).

Now consider solutions $D_{m} D_{s}^{+} g=a g$. If $a>0$, then the desired conclusion follows from (i). If $\alpha=0$, then this is a special case of $D_{m} D_{s}^{+} g=\alpha$ already con- 
sidered. Let $\alpha<0$. Then $u(x, \alpha)$ and $v(x, \alpha)$ are two linearly independent solutions since $D_{s}^{+} u(0, \alpha)=0, D_{s}^{+} v(0, \alpha)=1$ and $u(0, \alpha)=1$. Clearly $|u(x, \alpha)| \leq$ $u(x,-\alpha),|v(x, \alpha)| \leq|v(x,-\alpha)|$. Since $-\alpha>0$ and $u(x,-\alpha), v(x,-\alpha)$ are solu. tions of $D_{m} D_{s}^{+} g=(-a) g$, the desired conclusion follows from (i).

We now prove (iii). Consider solutions of the equation $D_{m} D_{s}^{+} f+\partial f / \partial t=0$ of the form $f(x, t)=t^{n} \phi_{0}(x)+t^{n-1} \phi_{1}(x)+\cdots+\phi_{n}(x)$. We have for all $t \geq 0, x \in$ $\left(r_{0}, r_{1}\right)$,

$$
t^{n} D_{m} D_{s}^{+} \phi_{0}(x)+t^{n-1}\left(n \phi_{0}(x)+D_{m} D_{s}^{+} \phi_{1}(x)\right)+\cdots+\left(\phi_{n-1}(x)+D_{m} D_{s}^{+} \phi_{n}(x)\right)=0 \text {. }
$$

Therefore $D_{m} D_{s}^{+} \phi_{0}(x)=0$, and in general for $1 \leq i \leq n, D_{m} D_{s}^{+} \phi_{i}(x)=$ $-(n-i+1) \phi_{i-1}(x)$. Hence there exist arbitrary constants $c_{0}, d_{0}, c_{1}, d_{1}, \ldots, c_{n}$, $d_{n}$ such that

$$
\phi_{0}(x)=c_{0}+d_{0} s(x)
$$

and for $1 \leq i \leq n$,

$$
\phi_{i}(x)=-(n-i+1)\left[\int_{0}^{x} \int_{0}^{y} \phi_{i-1}(z) d m(z) d s(y)+c_{i}+d_{i} s(x)\right] .
$$

It is easy to see by induction that $\phi_{i}$ is a linear combination of $u_{0}, \cdots, u_{i}$, $v_{0}, \cdots, v_{i}$. Therefore by Lemmas 7 and 8 , given $\lambda>0$, there exist constants $K_{1}$, $K_{2}$ such that, for $i=0, \cdots, n,\left|\phi_{i}(x)\right| \leq K_{1} u(x, \lambda)+K_{2}|v(x, \lambda)|$. Hence the desired conclusion follows from (i). Q.E.D.

From (iii) of Theorem 5 , it follows that $t^{n} \phi_{0}(X(t))+t^{n-1} \phi_{1}(X(t))+\cdots+$ $\phi_{n}(X(t)), t \geq 0$, is a martingale, where $\phi_{0}, \cdots, \phi_{n}$ are given by $(13 \mathrm{a}, \mathrm{b})$. We shall call such martingales time-polynomial martingales, since they are polynomials of the time variable. By first setting $c_{0}=(-1)^{n} / n !, c_{1}=\cdots=c_{n}=$ $d_{0}=\cdots=d_{n}=0$, and then setting $d_{0}=(-1)^{n} / n !, d_{1}=\cdots=d_{n}=c_{0}=\cdots=$ $c_{n}=0$, one obtains the two kinds of time-polynomial martingales of degree $n$ discussed by Arbib [1] in the case of a regular diffusion with generator $D_{m} D^{+}$and natural boundaries at $\pm \infty$. Arbib's proof depends on the direct evaluation of $E_{0}\left[\phi_{i}(X(t))\right]$ which in turn makes use of the behavior of the positive decreasing and increasing solutions of $D_{m} D^{+} g=a g$ at the boundaries $+\infty$ and $-\infty$ respectively. Arbib [1] has obtained a characterization of the diffusion with generator $D_{m} D^{+}$and natural boundaries $\pm \infty$ in terms of time-polynomial martingales. In our present case, in order that a stochastic process $X(t), t \geq 0$, taking values in $\left(r_{0}, r_{1}\right)$ be a regular diffusion with generator $D_{m} D_{s}^{+}$and natural boundaries at $r_{0}$, $r_{1}$, it is both necessary and sufficient that almost all sample paths of $X(t)$ are continuous, and

are both martingales.

$$
\{s(X(t)), t \geq 0\}, \quad\left\{\int_{0}^{X(t)} m(0, y] d s(y)-t, t \geq 0\right\}
$$


Under certain assumptions on the diffusion coefficients $\sigma(x)>0$ and $\mu(x)$, a standard Ito process $X(t)$ on the real line can be considered as a regular diffusion with generator $D_{m} D_{s}^{+}$and natural boundaries at $\pm \infty$, where

$$
s(x)=\int_{0}^{x} e^{-B(y)} d y, \quad m(x)=\int_{0}^{x} \frac{2 e^{B(y)}}{\sigma^{2}(y)} d y, \quad B(x)=\int_{0}^{x} \frac{2 \mu(y)}{\sigma^{2}(y)} d y .
$$

In such cases, by (ii) of Theorem 5 , for any real number $\alpha$, if

$$
\frac{\sigma^{2}(x)}{2} \frac{d^{2} g}{d x^{2}}+\mu(x) \frac{d g}{d x}=\alpha g
$$

then $e^{-a t} g(X(t)), t \geq 0$, is a martingale, while if

$$
\frac{\sigma^{2}(x)}{2} \frac{d^{2} g}{d x^{2}}+\mu(x) \frac{d g}{d x}=\alpha
$$

then $g(X(t))-a t, t \geq 0$, is a martingale (cf. Doob [2]).

Let us turn to the case where $r_{0}, r_{1}$ are both inaccessible, but not both natural. Let $X(t)$ be the Bessel process of order $m \geq 2$, i.e., $X(t)$ has transition density

$$
p(t, x, y)=t^{-1}(x y)^{1-m / 2} y^{m-1} I_{m / 2-1}(x y / t) \exp \left(-\left(x^{2}+y^{2}\right) / 2 t\right), \quad t>0, x, y>0 .
$$

$\left(I_{r}(z)\right.$ denotes the modified Bessel function of the first kind.) Here 0 is an entrance boundary and $\infty$ is a natural boundary. In [8], it is shown that for the increasing solution

$$
g_{1}(x)=x^{1-m / 2} I_{m / 2-1}(\sqrt{2 \alpha} x)
$$

of

$$
\frac{1}{2}\left(\frac{d^{2} g}{d x^{2}}+\frac{m-1}{x} \frac{d g}{d x}\right)=\alpha g \quad(\alpha>0),
$$

$e^{-a t} g_{1}(X(t)), t \geq 0$, is a martingale, whereas the decreasing solution $g_{2}(x)=$ $x^{1-m / 2} K_{m / 2-1}(\sqrt{2 a} x)\left(K_{r}(z)\right.$ being the modified Bessel function of the second kind) fails to give a martingale. In the case $\alpha<0$, the second order differential equation above has two linearly independent solutions, one of which is $\tilde{g}_{1}(x)=$ $x^{1-m / 2} J_{m / 2-1}(\sqrt{2|a|} x)$, where $J_{r}(z)$ is the Bessel function of the first kind. The other linearly independent solution $\widetilde{g}_{2}(x)$ is $x^{1-m / 2} J_{1-m / 2}(\sqrt{2|a|} x)$ if $m \neq 2,4$, $6, \ldots$, and is $x^{1-m / 2} Y_{m / 2-1}(\sqrt{2 \mid a} x)$ if $m=2,4,6, \ldots$, where $Y_{r}(z)$ is the Bessel function of the second kind. It is easy to see that $e^{-a t} \tilde{g}_{1}(X(t)), t \geq 0$, is a martingale, while $e^{-a t \tilde{g}_{2}}(X(t)), t \geq 0$, is not a martingale. For $\alpha=0$, two linearly independent solutions of the above second order differential equation are $g_{1}^{*}=1$ and 


$$
g_{2}^{*}(x)= \begin{cases}-x^{2-m} & (m>2) \\ \log x & (m=2)\end{cases}
$$

Clearly $g_{1}^{*}(X(t)), t \geq 0$, is a martingale. However $g_{2}^{*}(X(t)), t \geq 0$, is not a martingale. The function $g_{2}^{*}$ is the scale of $X(t)$. Such a phenomenon is actually common to all one-dimensional diffusions with an entrance boundary, as we shall show below.

Let $X(t)$ be a conservative regular strong Markov process on $\left(r_{0}, r_{1}\right)$ such that $r_{0}$ is an entrance boundary. Again for notational convenience, assume that $0 \in\left(r_{0}, r_{1}\right), s(0)=m(0)=m(0-)=0$, and we define $u(x, \alpha)$ as in (7), $v(x, \alpha)$ as in (8), $u_{+}(x, \lambda), u_{-}(x, \lambda)$ for $\lambda>0$ as in (10). Since $r_{0}$ is an entrance boundary, $m\left(r_{0}\right)$ is finite, but $s\left(r_{0}\right)=-\infty$. Also $\int_{r_{0}}^{0}|s(z)| d m(z)<\infty$.

Lemma 9. Assume that $r_{0}$ is an entrance boundary, $0 \in\left(r_{0}, r_{1}\right), s(0)=m(0)$ $=m\left(0_{-}\right)=0$. For $x \in\left(r_{0}, 0\right)$, define $\psi_{1}(x)=-m(x)$, and for $n \geq 1$,

$$
\psi_{n+1}(x)=\int_{x}^{0} \int_{y}^{0} \psi_{n}(z) d s(z) d m(y) .
$$

Also define

$$
\omega_{1}(x)=-\int_{x}^{0} s(z) d m(z)
$$

and for $n \geq 1$,

$$
\omega_{n+1}(x)=\int_{x}^{0} \int_{y}^{0} \omega_{n}(z) d s(z) d m(y) .
$$

Then for $n \geq 1, x \in\left(r_{0}, 0\right)$,

$$
\begin{aligned}
& 0<\psi_{n}(x) \leq\left(\left(\omega_{1}(x)\right)^{n-1} /(n-1) !\right) \psi_{1}(x) \\
& 0<\omega_{n}(x) \leq\left(\omega_{1}(x)\right)^{n} / n !
\end{aligned}
$$

Furthermore, $\lim _{x\rfloor_{0} r_{0}} \psi_{n}(x), \lim _{x\rfloor r_{0}} \omega_{n}(x)$ exist and are finite positive numbers. Define $\psi_{n}\left(r_{0}\right)=\lim _{x\left\lfloor r_{0}\right.} \psi_{n}(x), \omega_{n}\left(r_{0}\right)=\lim _{x\left\lfloor r_{0}\right.} \omega_{n}(x), n=1,2, \ldots$ Then the series $\sum_{n=1}^{\infty} \alpha^{n} \psi_{n}\left(r_{0}\right), \sum_{n=1}^{\infty} \alpha^{n} \omega_{n}\left(r_{0}\right)$ both converge for any real $\alpha$.

Proof. The inequality (14) clearly holds for $n=1$. Assume that it holds for $n$. Then for $x \in\left(r_{0}, 0\right)$,

$$
\begin{aligned}
\psi_{n+1}(x) & \leq \int_{x}^{0} \int_{y}^{0}\left(\omega_{1}(z)\right)^{n-1}|m(z)| d s(z) d m(y) /(n-1) ! \\
& \leq \frac{|m(x)|}{(n-1) !} \int_{x}^{0}\left(\omega_{1}(y)\right)^{n-1} \int_{y}^{0} d s(z) d m(y)=\left(\omega_{1}(x)\right)^{n} \psi_{1}(x) / n ! .
\end{aligned}
$$

Similarly we can prove (15). Clearly the functions $\psi_{n}(x), \omega_{n}(x)$ are decreasing for $x<0$, and so $\lim _{x \mid r_{0}} \psi_{n}(x), \lim _{x\left\lfloor r_{0}\right.} \omega_{n}(x)$ exist and are positive. Since (14) 
holds for all $x \in\left(r_{0}, 0\right)$, it follows that

$$
\psi_{n}\left(r_{0}\right) \leq\left(\omega_{1}\left(r_{0}\right)\right)^{n-1} \psi_{1}\left(r_{0}\right) /(n-1) !<\infty .
$$

Therefore $\sum_{n=1}^{\infty} a^{n} \psi_{n}\left(r_{0}\right)$ converges for all real $a$. The assertions for $\omega_{n}\left(r_{0}\right)$ are proved similarly.

Lemma 10. With the same assumptions as in Lemma 9, and $u_{n}(x), v_{n}(x)$ being defined by (7), (8), we have the following equalities for all $x \in\left(r_{0}, 0\right)$ and $n \geq 1$ :

$$
u_{n}(x)=\int_{x}^{0} \psi_{n}(y) d s(y), \quad v_{n}(x)=-\int_{x}^{0} \omega_{n}(y) d s(y) .
$$

Therefore $u_{n}(x) \leq \psi_{n}\left(r_{0}\right)|s(x)|$ and $\left|v_{n}(x)\right| \leq \omega_{n}\left(r_{0}\right)|s(x)|$. As $x \downarrow r_{0}, u_{n}(x) \sim$ $\psi_{n}\left(r_{0}\right)|s(x)|$ and $v_{n}(x) \sim \omega_{n}\left(r_{0}\right) s(x)$.

Proof. It is easy to see by induction that, for $n \geq 1, u_{n}(x)=\int_{x}^{0} \psi_{n}(y) d s(y)$. From this, it follows that $u_{n}(x) \leq \psi_{n}\left(r_{0}\right)|s(x)|$. On the other hand, given $\epsilon>0$, we can choose $a_{0} \in\left(r_{0}, 0\right)$ such that, if $r_{0}<x \leq a_{0}$, then $\psi_{n}(x)>\psi_{n}\left(r_{0}\right)-\epsilon$. For $x \in\left(r_{0}, a_{0}\right)$,

$$
u_{n}(x) \geq \int_{x}^{a_{0}} \psi_{n}(y) d s(y) \geq\left(\psi_{n}\left(r_{0}\right)-\epsilon\right)\left(s\left(a_{0}\right)-s(x)\right) .
$$

Therefore $\lim \inf _{x ! r_{0}} u_{n}(x) /|s(x)| \geq \psi_{n}\left(r_{0}\right)-\epsilon$. Since $\epsilon$ is arbitrary, $u_{n}(x) \sim$ $\psi_{n}\left(r_{0}\right)|s(x)|$ as $x \downarrow r_{0}$. The assertions for $v_{n}(x)$ can be proved similarly. Q.E.D.

Lemma 11. With the same assumptions as in Lemma 9, for any real number $a$, the series $\sum_{n=1}^{\infty} \alpha^{n} \psi_{n}\left(r_{0}\right), \sum_{n=1}^{\infty} a^{n} \omega_{n}\left(r_{0}\right)$ are convergent, and so we can define

$$
H(\alpha)=\sum_{n=1}^{\infty} \alpha^{n} \psi_{n}\left(r_{0}\right), \quad K(\alpha)=1+\sum_{n=1}^{\infty} \alpha^{n} \omega_{n}\left(r_{0}\right) .
$$

Then

$$
\lim _{\left.x\right|_{r_{0}}} u(x, \alpha) /|s(x)|=H(\alpha), \quad \lim _{x\rfloor_{0}} v(x, \alpha) / s(x)=K(\alpha)
$$

Proof. Since $\sum_{n=1}^{\infty}|\alpha|^{n} \psi_{n}\left(r_{0}\right)$ converges by Lemma 9, given $\epsilon>0$, we can choose $n_{0}$ such that $\sum_{n=n_{0}+1}^{\infty}|\alpha|^{n} \psi_{n}\left(r_{0}\right)<\epsilon$. By Lemma 10 , for any $x \in\left(r_{0}, 0\right)$, $u_{n}(x) \leq \psi_{n}\left(r_{0}\right)|s(x)|$, and so

$$
\left|\sum_{n=n_{0}+1}^{\infty} a^{n} u_{n}(x)\right|<\epsilon|s(x)| .
$$

Moreover, Lemma 10 implies

$$
\lim _{x\left\lfloor r_{0}\right.}\left(1+\alpha u_{1}(x)+\cdots+\alpha^{n} u_{n_{0}}(x)\right) /|s(x)|=\sum_{n=1}^{n_{0}} \alpha^{n} \psi_{n}\left(r_{0}\right) .
$$


Since $H(\alpha)-\epsilon \leq \sum_{n=1}^{n} \alpha^{n} \psi_{n}\left(r_{0}\right) \leq H(\alpha)+\epsilon$, it follows from (17) and (18) that

$$
H(\alpha)+2 \epsilon \geq \lim _{x\rfloor r_{0}} \sup \frac{u(x, \alpha)}{|s(x)|} \geq \underset{x_{x \mid r_{0}}}{\operatorname{limf}} \frac{u(x, \alpha)}{|s(x)|} \geq H(\alpha)-2 \epsilon .
$$

But $\epsilon$ is arbitrary, and so $\lim _{x\rfloor r_{0}} u(x, \alpha) /|s(x)|=H(\alpha)$. The proof for $v(x, \alpha)$ is similar. Q.E.D.

Lemma 12. With the same assumptions as in Lemma 9, if $\lambda>0$, then $H(\lambda)>$ 0 and as $a \downarrow r_{0}$,

$$
|s(a)| \sim u_{+}(a, \lambda) /\left(H(\lambda) \int_{r_{0}}^{r_{1}} \frac{d s(y)}{(u(y, \lambda))^{2}}\right) .
$$

Proof. Since $\psi_{n}\left(r_{0}\right)>0$ for all $n$, it is obvious that if $\lambda>0$, then $H(\lambda)>0$. By Lemma 11, $u(a, \lambda) \sim H(\lambda)|s(a)|$ as $a \downarrow r_{0}$. Since $\int_{r_{0}}^{r_{1}} d s(y) /(u(y, \lambda))^{2}<\infty$, the desired conclusion follows. Q.E.D.

Lemma 13. With the same assumptions as in Lemma 9,

(i) If $f(a)=o(s(a))$ as $a \downarrow r_{0}$, then given any $x \in\left(r_{0}, r_{1}\right)$ and $t>0$,

$$
\lim _{a\rfloor_{0}} f(a) P_{x}\left[\xi_{a}<t\right]=0 .
$$

(ii) Given any $x \in\left(r_{0}, r_{1}\right)$, there exists $t>0$ such that

$$
\underset{a ! r_{0}}{\lim \sup _{0}}|s(a)| P_{x}\left[\xi_{a}<t\right]>0 .
$$

Proof. (i) We fix $\lambda>0$ and note that, given any $t>0, x \in\left(r_{0}, r_{1}\right)$,

$$
\begin{aligned}
|f(a)| P_{x}\left[\xi_{a}<t\right] & \leq e^{\lambda t}|f(a)| \int_{0}^{\infty} e^{-\lambda \tau} d P_{x}\left[\xi_{a}<\tau\right] \\
& =e^{\lambda t}|f(a)| u_{+}(x, \lambda) / u_{+}(a, \lambda) \quad \text { if } a<x .
\end{aligned}
$$

Since $f(a)=o(s(a))$ and $s(a)=O\left(u_{+}(a, \lambda)\right)$, (i) follows immediately.

(ii) Given $x \in\left(r_{0}, r_{1}\right)$, we take any $\lambda>0$ and define

$$
F_{a}(t)=|s(a)| \int_{0}^{t} e^{-\lambda \tau} d P_{x}\left[\xi_{a}<\tau\right], \quad t \geq 0 .
$$

$F_{a}$ is a continuous increasing function on $[0, \infty)$ with $F_{a}(0)=0$. By Lemma 12, there exist a constant $c>0$ and $a_{0} \in\left(r_{0}, x \wedge 0\right)$ such that $|s(a)| \leq$ $c u_{+}(a, \lambda) / u_{+}(x, \lambda)$ for all $a \in\left(r_{0}, a_{0}\right)$. Now if $a \in\left(r_{0}, a_{0}\right)$,

$$
\int_{0}^{\infty} d F_{a}=|s(a)| \frac{u_{+}(x, \lambda)}{u_{+}(a, \lambda)} \leq c
$$

and so $F_{a}$ defines a measure on $[0, \infty)$ with total mass $\leq c$. By the Helly-Bray Lemma, 


$$
\lim _{a\left\lfloor_{0}\right.} F_{a}(t)=0 \quad \forall t \in[0, \infty) \quad \Rightarrow \lim _{a !_{0}} \int_{0}^{\infty} e^{-a t} d F_{a}(t)=0 \quad \forall a>0
$$

Take any $a>0$. For $a \in\left(r_{0}, a_{0}\right)$,

$$
\begin{aligned}
\int_{0}^{\infty} e^{-a t} d F_{a}(t) & =|s(a)| \frac{u_{+}(x, a+\lambda)}{u_{+}(a, a+\lambda)} \\
& \rightarrow u_{+}(x, a+\lambda) /\left(H(\alpha+\lambda) \int_{r_{0}}^{r_{1}} \frac{d s(y)}{(u(y, a+\lambda))^{2}}\right) \text { as } a \downarrow r_{0} .
\end{aligned}
$$

The last relation follows from Lemma 12. Since $F_{a}(0)=0$ for all $a$, we can therefore find $t_{0} \in(0, \infty)$ such that $F_{a}\left(t_{0}\right)$ does not converge to 0 as $a \downarrow r_{0}$, i.e., $\lim \sup _{\left.a\right|_{r_{0}}} F_{a}\left(t_{0}\right)>0$. But $F_{a}\left(t_{0}\right) \leq|s(a)| P_{x}\left[\xi_{a}<t_{0}\right]$ and so $\lim \sup _{a i_{0}}|s(a)| P_{x}\left[\xi_{a}<t_{0}\right]>0$. Q.E.D.

Theorem 6. Let $X(t)$ be a conservative regular continuous strong Markov process on an interval $I$ whose endpoints are $r_{0}, r_{1}$, where $-\infty \leq r_{0}<r_{1} \leq \infty$. Let $s(x)$ be the scale and $m$ the speed measure of $X(t)$.

(i) Suppose $r_{0}, r_{1}$ are inaccessible. If $f:\left(r_{0}, r_{1}\right) \times[0, \infty) \rightarrow(-\infty, \infty)$ is continuous, $\partial f / \partial t$ is also continuous and $D_{m} D_{s}^{+} f$ exists and satisfies $\partial f / \partial t+$ $D_{m} D_{s}^{+} f=0$ on $\left(r_{0}, r_{1}\right) \times[0, \infty)$, then $f$ is parabolic for $X(t)$. Suppose furthermore that for $i=0,1$ and any $T>0$,

(a) $\max _{0 \leq t \leq T}|f(x, t)|=o(s(x))$ as $x \rightarrow r_{i}$, if $r_{i}$ is an entrance boundary and

(b) there exists $\alpha_{T}>0$ and a function $g_{T}:\left(r_{0}, r_{1}\right) \rightarrow(-\infty, \infty)$ satisfying $D_{m} D_{s}^{+} g_{T}=\alpha_{T} g_{T}$ such that $\max _{0 \leq t \leq T}|f(x, t)|=O\left(\left|g_{T}(x)\right|\right)$ as $x \rightarrow r_{i}$, if $r_{i}$ is a natural boundary.

Then $f(x(t), t+r), t \geq 0$, is a martingale with respect to $P_{x}$ for all $x \in$ $\left(r_{0}, r_{1}\right)$ and all $r \geq 0$.

(ii) If $r_{0}$ is an entrance boundary and $g$ is a positive decreasing solution of $D_{m} D_{s}^{+} g=\lambda g$ with $\lambda>0$, then $\lim _{x \mid r_{0}} g(x) /|s(x)|$ exists and is positive, and $\{s(X(t)), t \geq 0\},\left\{e^{-\lambda t} g(X(t)), t \geq 0\right\}$ are not martingales with respect to $P_{x}$ for any $x \in\left(r_{0}, r_{1}\right)$. A similar assertion (replacing 'positive drecreasing solution' in the above by 'positive increasing solution') bolds if $r_{1}$ is an entrance boundary.

(iii) Suppose $r_{0}$ is an entrance boundary and $r_{1}$ is a natural boundary. If $b$ is a positive increasing solution of $D_{m} D_{s}^{+} b=\lambda b$ with $\lambda>0$, then $e^{-\lambda t} b(X(t))$, $t \geq 0$, is a martingale with respect to $P_{x}$ for all $x \in\left(r_{0}, r_{1}\right)$. Let $\alpha<0$. For notational convenience, assume $0 \in\left(r_{0}, r_{1}\right), s(0)=m(0)=m(0-)=0$, and define $H(\alpha), K(\alpha)$ by (16). If $H(\alpha)$ and $K(\alpha)$ are not both zero, then the vector space of all solutions $g$ of $D_{m} D_{s}^{+} g=$ ag such that $e^{-a t} g(X(t)), t \geq 0$, is a martingale with respect to $P_{x}$ for any $x \in\left(r_{0}, r_{1}\right)$ bas dimension 1 . If $H(\alpha)$ and $K(\alpha)$ are both equal to zero, then, for any solution $g$ of $D_{m} D_{s}^{+} g=a g, e^{-a t} g(X(t)), t \geq 0$, is a 
martingale with respect to $P_{x}$ for any $x \in\left(r_{0}, r_{1}\right)$.

Proof. We may without loss of generality assume throughout that $0 \in\left(r_{0}, r_{1}\right)$, $s(0)=m(0)=m\left(0_{-}\right)=0$.

(i) Suppose $r_{0}, r_{1}$ are both inaccessible. If $f:\left(r_{0}, r_{1}\right) \times[0, \infty) \rightarrow(-\infty, \infty)$ is continuous, $\partial f / \partial t$ is also continuous and $D_{m} D_{s}^{+} f$ exists and satisfies $\partial f / \partial t+$ $D_{m} D_{s}^{+} f=0$ on $\left(r_{0}, r_{1}\right) \times[0, \infty)$, then we have already proved that $f$ is parabolic for $X(t)$. Let $r \geq 0, t \geq 0$. If $r_{0}$ is an entrance boundary, then it follows from condition (a) and Lemma 13(i) that, for $x \in\left(r_{0}, r_{1}\right), r_{0}<a<b<r_{1}$,

$$
\int_{\left[\xi_{a^{<r} \wedge} \wedge \xi_{b}\right]}\left|f\left(a, t+\xi_{a}\right)\right| d P_{x} \leq\left(\max _{0 \leq \sigma \leq t+r}|f(a, \sigma)|\right) P_{x}\left[\xi_{a}<r\right] \rightarrow 0 \text { as } a \downarrow r_{0} .
$$

If $r_{0}$ is a natural boundary, then it follows from condition (b) and Lemma 6 that

$$
\int_{\left[\xi_{a^{<}<} \wedge \xi_{b}\right]}\left|f\left(a, t+\xi_{a}\right)\right| d P_{x}^{0} \leq C\left|g_{T}(a)\right| \cdot P_{x}\left[\xi_{a}<r\right] \rightarrow 0 \text { as } a \downarrow r_{0} .
$$

Similarly we can prove that, under conditions (a) and (b),

$$
\lim _{b \mid r_{1}} \int_{\left[\xi_{b \leq r} \wedge \xi_{a}\right]}\left|f\left(b, t+\xi_{b}\right)\right| d P_{x}=0
$$

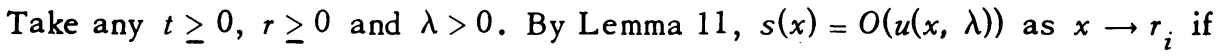
$r_{i}$ is an entrance boundary. Therefore conditions (a) and (b) imply that there exist $K$ such that $|f(x, t+r)| \leq K u(x, \lambda)$ for all $x \in\left(r_{0}, r_{1}\right)$. Now $u(x, \lambda) \geq$ $e^{-\lambda r} \int u(y, \lambda) P(r, x, d y)$. Therefore $\int|f(y, t+r)| P(r, x, d y)<\infty$, and by the dominated convergence theorem,

$$
\lim _{a \mid r 0, b r_{1}} \int_{\left[\xi_{a b \geq r]}\right.} f(X(r), t+r) d P_{x}=\int f(y, t+r) P(r, x, d y) .
$$

Hence by Theorem 4, $f(X(t), t+r), t \geq 0$, is a martingale with respect to $P_{x}$ for all $x \in\left(r_{0}, r_{1}\right)$ and $r \geq 0$.

(ii) Since $g$ is a positive decreasing solution of $D_{m} D_{s}^{+} g=\lambda g, g(x)=$ $c_{1} u_{+}(x, \lambda)+c_{2} u_{-}(x, \lambda)$ with $c_{1}>0$. (Note that $c_{2}$ must vanish if $r_{1}$ is also an inaccessible boundary.) Therefore by Lemma 12 ,

$$
\lim _{x \backslash r_{0}} g(x) /|s(x)|=\lim _{x\left\lfloor r_{0}\right.} c_{1} u_{+}(x, \lambda) /|s(x)|
$$

indeed exists and is positive.

Take any $x \in\left(r_{0}, r_{1}\right)$. By Lemma 13, there exists $t_{0}>0$ such that $\lim \sup _{a ! r_{0}}|s(a)| P_{x}\left[\xi_{a}<t_{0}\right]>0$. Therefore we also have lim $\sup _{a r_{0}} g(a) P_{x}\left[\xi_{a}<t_{0}\right]$ $>0$. Apply Theorem 4 with $f(z, t)$ equal to $s(z)$ or $e^{-\lambda t} g(z)$. Then condition (i) of that theorem is violated and therefore $\{s(X(t)), t \geq 0\}$ and $\left\{e^{-\lambda t} g(X(t)), t \geq 0\right\}$ cannot be martingales with respect to $P_{x}$.

(iii) If $b$ is a positive increasing solution of $D_{m} D_{s}^{+} b=\lambda b$ with $\lambda>0$, then 
$f(x, t)=e^{-\lambda t} b(x)$ satisfies conditions (a), (b) of (i), and so $e^{-\lambda t} b(X(t)), t \geq 0$, is a martingale with respect to $P_{x}$ for all $x \in\left(r_{0}, r_{1}\right)$.

Take $\alpha<0$. Suppose $H(\alpha) \neq 0$, By Lemma 11, $u(a, \alpha) \sim H(\alpha)|s(a)|$ as $a \downarrow r_{0}$. Therefore given $x \in\left(r_{n}, r_{1}\right)$, there exists $t_{n}>0$ such that

$$
\underset{\quad \lim \sup _{0}}{\log }|u(a, a)| P_{x}\left[\xi_{a}<t_{0}\right]>0,
$$

and $e^{-a_{t}} u(X(t), \alpha), t \geq 0$, is not a martingale with respect to $P_{x}$ for any $x \in\left(r_{n}, r_{1}\right)$.

Assume for definiteness that $H(\alpha)>0$. (The case $H(\alpha)<0$ is treated similarly.) Then $u(a, a) \sim H(\alpha)|s(a)|$ as $a \downarrow r_{0}$. Therefore we can choose $c>0$ and $x_{0}<0$ such that $u(y, \alpha) \geq c|s(y)|$ on $\left(r_{0}, x_{0}\right]$. We can then define $u_{(\alpha)}:\left(r_{0}, x_{0}\right)$ $\rightarrow(-\infty, \infty)$ by

$$
u_{(\alpha)}(x)=u(x, \alpha) \int_{r_{0}}^{x} \frac{d s(y)}{(u(y, \alpha))^{2}} .
$$

It can be proved that $D_{m} D_{s}^{+} u_{(\alpha)}(x)=\alpha u_{(\alpha)}(x), x \in\left(r_{0}, x_{0}\right)$ (cf. pp. 27-28 of [9]). Since $u(x, \alpha), v(x, \alpha)$ are two linearly independent solutions of $D_{m} D_{s}^{+} g=\alpha g$ on $\left(r_{0}, x_{0}\right)$, there exist unique constants $c_{1}, c_{2}$ such that

$$
u_{(\alpha)}(x)=c_{1} u(x, \alpha)+c_{2} v(x, \alpha), \quad x \in\left(r_{0}, x_{0}\right) .
$$

Let $w(x)=c_{1} u(x, \alpha)+c_{2} v(x, \alpha), x \in\left(r_{0}, r_{1}\right)$. Then $D_{m} D_{s}^{+} w=\alpha w$ on $\left(r_{0}, r_{1}\right)$. It is easy to see that

$$
\lim _{x\left\lfloor r_{0}\right.} w(x)=\lim _{x \mathfrak{r}_{0}} u_{(\alpha)}(x)=1 / H(\alpha) .
$$

From this, it is clear that $u(x, \alpha)$ and $w(x)$ are linearly independent. Also the function $e^{-a t} w(x)$ satisfies conditions (a), (b) of (i) and so $e^{-a t} w(X(t)), t \geq 0$, is a martingale with respect to $P_{x}$ for all $x \in\left(r_{0}, r_{1}\right)$.

Let $g$ be any solution of $D_{m} D_{s}^{+} g=\alpha g$ such that $e^{-\alpha t} g(X(t)), t \geq 0$, is a martingale with respect to $P_{x}$ for any $x \in\left(r_{0}, r_{1}\right)$. Then $g(x)=K_{1} u(x, \alpha)+$ $K_{2} w(x)$. If $K_{1} \neq 0, \lim _{x \mid r_{0}} g(x) /|s(x)|=K_{1} H(\alpha) \neq 0$, and so $e^{-\alpha t} g(X(t)), t \geq 0$, cannot be a martingale with respect to $P_{x}$ for any $x \in\left(r_{0}, r_{1}\right)$, contradicting the assumption on $g$. Therefore $K_{1}=0$ and $g$ is a scalar multiple of $w$.

The case $K(\alpha) \neq 0$ can be treated in an analogous way.

Now suppose that $H(\alpha)=0$ and $K(\alpha)=0$. Then by Lemma 11, $u(a, \alpha)=$ $o(s(a))$ as $a \downarrow r_{0}$ and $v(a, \alpha)=o(s(a))$ as $a \downarrow r_{0}$. Therefore for any solution $g$ of $D_{m} D_{s}^{+} g=\alpha g, g(a)=o(s(a))$ as $a \downarrow r_{0}$. Hence the function $f(x, t)=e^{-\lambda t} g(x)$ satisfies conditions (a), (b) of (i) and so $e^{-\lambda t} g(X(t)), t \geq 0$, is a martingale with respect to $P_{x}$ for all $x \in\left(r_{0}, r_{1}\right)$. Q.E.D.

5. Application to boundary crossing probabilities. Let $\left(Y(t), \zeta, \mathcal{F}_{t}^{s}, P_{s, x}\right)$ be a continuous normal strong Markov process on a locally compact, second count- 
able, Hausdorff space $M$. Let $Z(t)$ be the corresponding space-time process. For any open subset $G$ of $M \times[0, \infty)$, set

$$
\psi(x, s)=P_{s, x}[(Y(t), t) \notin G \text { for some } s \leq t<\zeta] .
$$

The function $\psi$ is parabolic for $Y(t)$ on $G$. In particular, let us consider the case where $Y(t)$ is the diffusion in Theorem 3. Then by that theorem, $\psi$ belongs to $C^{2,1}(G)$ and satisfies the partial differential equation $L \psi=0$ on $G$, where $L$ is the backward parabolic operator defined in (3).

Now suppose that $X(t)$ is a regular conservative continuous strong Markov process on $\left(r_{0}, r_{1}\right)$ with speed measure $m$ and scale function $s$ such that the boundaries $r_{0}, r_{1}$ are both inaccessible $\left(-\infty \leq r_{0}<r_{1} \leq \infty\right)$. For $\lambda>0$, let $f_{\lambda}^{(0)}$ be a positive decreasing solution and let $f_{\lambda}^{(1)}$ be a positive increasing solution of $D_{m} D_{s}^{+} f=\lambda f$. Let $F_{i}(i=0,1)$ be a nontrivial measure on $(0, \infty)$ such that $\phi_{i}(x, \tau)<\infty$ for all $x \in\left(r_{0}, r_{1}\right)$, where we define

$$
\phi_{i}(x, t)=\int_{0}^{\infty} e^{-\lambda t} f_{\lambda}^{(i)}(x) d F_{i}(\lambda) .
$$

If $r_{0}, r_{1}$ are both natural boundaries, then since $\lim _{x \mid r 1} f_{\lambda}^{(1)}(x)=\infty$ and $\lim _{x \downarrow r_{0}} f_{\lambda}^{(1)}(x)=0$, therefore given $\epsilon>0$, we have a unique solution $x=B_{1}(t, \epsilon)$, with $r_{0}<x<r_{1}$, of the equation $\phi_{1}(x, t)=\epsilon$ for any $t \geq \tau$. By our results in $\$ 4$, $\phi_{1}(X(t), t), t \geq \tau$, is a martingale with respect to $P_{x}$ for any $x \in\left(r_{0}, r_{1}\right)$. If $\phi_{1}(X(t), t)$ converges to 0 in probability as $t \rightarrow \infty$ on the event $\left[\sup _{t \geq T} \phi_{1}(X(t), t)\right.$ $<\epsilon]$, then it follows from [10] that

$$
\begin{aligned}
& P_{x}\left[X(t) \geq B_{1}(t, \epsilon) \text { for some } t \geq \tau\right] \\
& \quad=P_{x}\left[X(\tau) \geq B_{1}(\tau, \epsilon)\right]+\frac{1}{\epsilon} \int_{0}^{\infty} \int_{\left[X(\tau)<B_{1}(\tau, \epsilon)\right]} e^{-\lambda \tau} f_{\lambda}^{(1)}(X(\tau)) d P_{x} d F_{1}(\lambda) .
\end{aligned}
$$

Likewise given any $\epsilon>0$, there exists a unique solution $x=B_{0}(t, \epsilon)$ of the equation $\phi_{0}(x, t)=\epsilon$ for $t \geq \tau$. Also $\phi_{0}(X(t), t), t \geq \tau$, is a martingale $w$ ith respect to $P_{x}$ for any $x \in\left(r_{0}, r_{1}\right)$. If $\phi_{0}(X(t), t)$ converges to 0 in probability on $\left[\sup _{t \geq \tau} \phi_{0}(X(t), t)<\epsilon\right]$, then again it follows from [10] that

$$
\begin{aligned}
P_{x}[X(t) & \left.\leq B_{0}(t, \epsilon) \text { for some } t \geq \tau\right] \\
= & P_{x}\left[X(\tau) \leq B_{0}(\tau, \epsilon)\right]+\frac{1}{\epsilon} \int_{0}^{\infty} \int_{\left[X(\tau)>B_{0}(\tau, \epsilon)\right]} e^{-\lambda \tau} f_{\lambda}^{(0)}(X(\tau)) d P_{x} d F_{0}(\lambda) .
\end{aligned}
$$

When the boundaries are both inaccessible, but not both natural, we still have $\lim _{x \rightarrow r_{i}} f_{\lambda}^{(i)}(x)=\infty, i=0,1$. But $\lim _{x \rightarrow r_{0}} f_{\lambda}^{(1)}(x)$ may not be zero if $r_{0}$ is not natural. However, given any $\epsilon>\phi_{1}\left(r_{0}, \tau\right)$, we still have a unique solution $x=$ $B_{1}(t, \epsilon)$ of the equation $\phi_{1}(x, t)=\epsilon$ for any $t \geq \tau$. If $r_{1}$ is not natural, then $e^{-\lambda t} f_{\lambda}^{(1)}(X(t)), t \geq 0$, fails to be a martingale. However, the function $\phi_{1}(x, t)$ is parabolic for $X(t)$. Let $\epsilon>\phi_{1}\left(r_{0}, \tau\right)$. Take any $\epsilon^{\prime}>\epsilon$ and define $\sigma=$ inf $\left\{t \geq \tau \mid \phi_{1}(X(t), t) \geq \epsilon^{\prime}\right\}$. Then $\phi_{1}(X(t \wedge \sigma), t \wedge \sigma), t \geq \tau$, is a martingale with 
respect to $P_{x}$ for any $x \in\left(r_{0}, r_{1}\right)$. Therefore if $\phi_{1}(X(t), t)$ converges to 0 in probability as $t \rightarrow \infty$ on [ $\sup _{t \geq \tau} \phi_{1}(X(t), t)<\epsilon$ ], then it follows from [10] that

$$
\begin{aligned}
P_{x} & {\left[X(t) \geq B_{1}(t, \epsilon) \text { for some } t \geq \tau\right] } \\
& =P_{x}\left[\phi_{1}(X(t \wedge \sigma), t \wedge \sigma) \geq \epsilon \text { for some } t \geq \tau\right] \\
& =P_{x}\left[X(\tau) \geq B_{1}(\tau, \epsilon)\right]+\frac{1}{\epsilon} \int_{0}^{\infty} \int_{\left[X(\tau)<B_{1}(\tau, \epsilon)\right]} e^{-\lambda \tau} f_{\lambda}^{(1)}(X(\tau)) d P_{x} d F_{1}(\lambda) .
\end{aligned}
$$

In the case where $m\left(r_{1}\right)<\infty$ and $m\left(r_{0}\right)>-\infty$, the transition probabilities $P(t, x,$.$) converge weakly to a probability distribution on \left(r_{0}, r_{1}\right)$ whose distribution function $G(y)$ is $\left(m(y)-m\left(r_{0}\right)\right)\left(m\left(r_{1}\right)-m\left(r_{0}\right)\right)^{-1}$ as $t \rightarrow \infty$ (cf. [9]). It is obvious in this case that, for $i=0,1$, the martingale $e^{-\lambda t} f_{\lambda}^{(i)}(X(t))$ converges to zero in probability (and therefore almost surely by the martingale convergence theorem) as $t \rightarrow \infty$ for any initial state $x$. Suppose $F_{i}$ is any finite measure with bounded support on $(0, \infty)$. Then it easily follows that $\phi_{i}(X(t), t)$ converges to zero almost surely as $t \rightarrow \infty$ for any initial state $x$, and so (19), (20) and (21) can be applied to give the probability that $X(t)$ would ever cross certain moving boundaries.

\section{REFERENCES}

1. M. A. Arbib, Hitting and Martingale 'characterizations of one-dimensional diffusions, Z. Wahrscheinlichkeitstheorie und Verw. Gebeite 4 (1965), 232-247. MR 33 \#776.

2. J. L. Doob, Martingales and one-dimensional diffusion, Trans. Amer. Math. Soc. 78 (1955), 168-208. MR 17, 50 .

3. - A probability approach to the heat equation, Trans. Amer. Math. Soc. 80 (1955), 216-280. MR 18, 76.

4. E. B. Dynkin, Theory of Markov processes, Fizmatgiz, Moscow, 1959; English transl., Prentice-Hall, Englewood Cliffs, N. J.; Pergamon Press, Oxford, 1961. MR 24 \#A1745; \#A1747.

5. - Markov processes, Fizmatgiz, Moscow, 1963; English transl., Die Grundlehren der math. Wissenschaften, Bände 121, 122, Academic Press, New York; Springer-Verlag, Berlin, 1965. MR 33 \#1887.

6. K. Ito and H. P. McKean, Jr., Diffusion processes and their sample paths, Die Grundlehren der math. Wissenschaften, Band 125, Academic Press, New York; SpringerVerlag, Berlin, 1965. MR 33 \#8031.

7. A. Friedman, Partial differential equations of parabolic type, Prentice-Hall, Englewood Cliffs, N. J., 1964. MR $31 \# 6062$.

8. T. L. Lai, Martingales and boundary crossing probabilities for Markov processes (to appear).

9. P. Mandl, Analytical treatment of one-dimensional Markov processes, Die Grundlehren der math. Wissenschaften, Band 151, Springer-Verlag, New York, 1968. MR 40 \#930.

10. H. Robbins and D. Siegmund, Boundary crossing probabilities for the Wiener process and sample sums, Ann. Math. Statist. 41 (1970), 1410-1429.

DEPARTMENT OF MATHEMATICAL STATISTICS, COLUMBIA UNIVERSITY, NEW YORK, NEW YORK 10027 